\title{
PATCHING AND WEAK APPROXIMATION IN ISOMETRY GROUPS
}

\author{
EVA BAYER-FLUCKIGER AND URIYA A. FIRST
}

\begin{abstract}
Let $R$ be a semilocal principal ideal domain. Two algebraic objects over $R$ in which scalar extension makes sense (e.g. quadratic spaces) are said to be of the same genus if they become isomorphic after extending scalars to all completions of $R$ and its fraction field. We prove that the number of isomorphism classes in the genus of unimodular quadratic spaces over (not necessarily commutative) $R$-orders is always a finite power of 2 , and under further assumptions, e.g., that the order is hereditary, this number is 1 . The same result is also shown for related objects, e.g., systems of sesquilinear forms. A key ingredient in the proof is a weak approximation theorem for groups of isometries, which is valid over any (topological) base field, and even over semilocal base rings.
\end{abstract}

\section{INTRODUCTION}

Let $R$ be a semilocal principal ideal domain, or equivalently, a Dedekind domain with finitely many maximal ideals. For $\mathfrak{p} \in \operatorname{Spec} R$, let $R_{\mathfrak{p}}$ denote the localization of $R$ at $\mathfrak{p}$, and let $\hat{R}_{\mathfrak{p}}$ denote the $\mathfrak{p}$-adic completion of $R_{\mathfrak{p}}$. Note that $F:=\hat{R}_{0}$ is just the fraction field of $R$.

We define the genus of a quadratic form $q$ over $R$ to be the set of isomorphism classes of quadratic forms that become isomorphic to $q$ over $\hat{R}_{\mathfrak{p}}$ for all $\mathfrak{p} \in \operatorname{Spec} R$ (including $\mathfrak{p}=0$ ). This resembles the (much stronger) notion of genus of quadratic forms over the integers $[29, \S 102 \mathrm{~A}]$. A classical result states that the genus of integral quadratic forms is finite (see [29, Th. 102:8, Th. 103:4] and also [8, Th. 3.4, Th. 4.2] for generalizations).

Our notion of genus clearly generalizes to other objects defined over $R$ for which there is a notion of scalar extension. This paper is concerned with proving that the genus is finite for various types of objects of quadratic nature. Of particular interest are cases where the genus consists of a single isomorphism class, since then it is enough to check isomorphism over the completions $\left\{\hat{R}_{\mathfrak{p}}\right\}_{\mathfrak{p} \in \operatorname{Spec} R}$ in order to prove isomorphism over $R$. This can be regarded as algebraic patching of the kind that requires certain factorizations in the automorphism group. Such patching problems were considered by various authors, especially for torsors of group schemes; see for instance [28 and [20, §3], to name just a few examples.

Received by the editors April 7, 2015 and, in revised form, December 15, 2015.

2010 Mathematics Subject Classification. Primary 11E39, 11E41, $16 \mathrm{H} 10$.

Key words and phrases. Quadratic form, hermitian form, algebraic patching, weak approximation, genus, order, hereditary order, sesquilinear form, hermitian category.

The second-named author performed this research at EPFL, the Hebrew University of Jerusalem and the University of British Columbia (in this order), where he was supported by an SNFS grant \#IZK0Z2_151061, an ERC grant \#226135, and the UBC Mathematics Department, respectively. 
Let $A$ be an $R$-algebra endowed with a unitary $R$-algebra structure (all definitions are recalled in section (1). Then one can consider the genus of quadratic spaces over $A$. Assume henceforth that $A$ is finitely generated and torsion-free as an $R$-module, and let $(P,[f])$ be a unimodular quadratic space over $A$. We show that:

(1) $|\operatorname{gen}(P,[f])|$ is a finite power of 2 .

(2) If $A$ is hereditary, then $|\operatorname{gen}(P,[f])|=1$.

Recall that the algebra $A$ is hereditary if its one-sided ideals are projective. Notable examples of hereditary orders include maximal orders. We also bound the size of the genus in the non-hereditary case (see Theorem 5.11).

When $2 \in R^{\times}$, we extend the previous results to systems of sesquilinear forms and non-unimodular hermitian forms, using results from [5], [10] and [7. Specifically, we show that:

(3) Let $\left\{\sigma_{i}\right\}_{i \in I}$ be a family of $R$-involutions on $A$ and let $\left(P,\left\{f_{i}\right\}_{i \in I}\right)$ be a system of sesquilinear forms over $\left(A,\left\{\sigma_{i}\right\}_{i \in I}\right)$. Then $\left|\operatorname{gen}\left(P,\left\{f_{i}\right\}\right)\right|$ is a finite power of 2 .

(4) Let $\sigma: A \rightarrow A$ be an $R$-involution and let $u \in \operatorname{Cent}(A)$ be an element satisfying $u^{\sigma} u=1$. Assume that $A$ is hereditary. Then $|\operatorname{gen}(P, f)|=1$ for every $u$-hermitian space $(P, f)$ over $(A, \sigma)$ (unimodularity is not assumed).

As an application of (4), we show that Witt's Cancellation Theorem and a variant of Springer's Theorem hold for hermitian forms over involutary hereditary orders (unimodularity is not assumed).

A main tool in the proofs, which may be of interest in its own right, is a weak approximation theorem: Let $K$ be a topological commutative semilocal ring, let $F$ be a dense subring, let $A$ be a unitary $F$-algebra, and let $(P,[f])$ be a unimodular quadratic space over $A$. Denote by $O([f])$ the group of isometries of $[f]$, and let $\left[f_{K}\right]$ denote the scalar extension of $[f]$ to $A \otimes_{F} K$. Then, under mild assumptions, the closure of $O([f])$ in $O\left(\left[f_{K}\right]\right)$ is of finite index. Furthermore, if $F$ is a field, $K$ is a product of fields, and $O^{+}$denotes the connected component of $O([f])$ (when viewed as a group scheme), then $\overline{O^{+}(F)}=O^{+}(K)$.

Weak approximation theorems for adjoint algebraic groups over arbitrary topological fields were studied previously; see [36] and references therein (for instance). Our approach is somewhat different and relies on generalizations of Witt's Theorem to quadratic spaces over semilocal rings ([32, [17]).

We note that in our general setting $O([f])$ can indeed be regarded as an affine scheme over $\operatorname{Spec} F$, which is also smooth and faithfully flat. However, when $F$ is replaced with a commutative ring, some mild assumptions are necessary. For the sake of completeness, this is discussed in the appendix. The appendix is based on communications with Mathieu Huruguen, and we thank him for his contribution.

The paper is organized as follows: Section 1 recalls unitary rings and quadratic spaces. Section 2 deals with some topological issues required to phrase the weak approximation theorem, which is proved in section 3. In sections 4 and 5, we prove the finiteness of the genus, and in section [6 we characterize various cases in which the size of the genus is 1 . Finally, section 7 extends the previous results to systems of sesquilinear forms and non-unimodular hermitian forms. The cancellation and the variant of Springer's Theorem mentioned above are also proved there. In the appendix, we show that isometry groups can be regarded as smooth affine group schemes, provided mild assumptions. 


\section{Preliminaries}

This section recalls hermitian and quadratic forms over rings and various related notions. See [4] and 24] for an extensive discussion.

1A. Hermitian forms. Let $(A, \sigma)$ be a ring with involution and let $u \in \operatorname{Cent}(A)$ be an element satisfying $u^{\sigma} u=1$. Denote by $\mathscr{P}(A)$ the category of finitely generated projective right $A$-modules. For $P \in \mathscr{P}(A)$, we make $P^{*}:=\operatorname{Hom}_{A}(P, A)$ into a right $A$-module by setting

$$
(\psi a) x=a^{\sigma}(\psi x) \quad \forall \psi \in P^{*}, a \in A, x \in P .
$$

Observe that $*: \mathscr{P}(A) \rightarrow \mathscr{P}(A)$ is a contravariant functor. For every morphism $f \in \operatorname{Hom}_{A}(P, Q)$, the dual $f^{*} \in \operatorname{Hom}_{A}\left(Q^{*}, P^{*}\right)$ is given by $f^{*} \psi=\psi \circ f\left(\psi \in P^{*}\right)$.

Every morphism $f \in \operatorname{Hom}_{A}\left(P, P^{*}\right)$ gives rise to a map $\tilde{f}: P \times P \rightarrow A$ given by

$$
\tilde{f}(x, y)=(f x) y \quad \forall x, y \in P .
$$

The map $\tilde{f}$ is biadditive and satisfies

$$
\tilde{f}(x a, y b)=a^{\sigma} \tilde{f}(x, y) b \quad \forall x, y \in P, a, b \in A .
$$

Conversely, it is easy to see that any biadditive map $\tilde{f}: P \times P \rightarrow A$ satisfying (1.1) is induced by a unique homomorphism $f \in \operatorname{Hom}_{A}\left(P, P^{*}\right)$. The map $\tilde{f}$ is called a sesquilinear form and the pair $(P, f)$ or $(P, \tilde{f})$ is called a sesquilinear space (over $(A, \sigma))$. We say that $(P, f)$ is unimodular if $f$ is an isomorphism.

There is a natural homomorphism $\omega_{P}: P \rightarrow P^{* *}$ given by

$$
\left(\omega_{P} x\right) \phi=(\phi x)^{\sigma} u \quad \forall x \in P, \phi \in P^{*} .
$$

It is well-known that $\omega_{P}$ is an isomorphism (when $P \in \mathscr{P}(A)$ ). Notice that $\omega_{P}$ depends on $u$. A $u$-hermitian space over $(A, \sigma)$ is a sesquilinear space $(P, f)$ such that $f=f^{*} \omega_{P}$. This is equivalent to

$$
\tilde{f}(x, y)=\tilde{f}(y, x)^{\sigma} u \quad \forall x, y \in P .
$$

Let $(P, f)$ and $\left(P^{\prime}, f^{\prime}\right)$ be sesquilinear spaces. An isometry from $(P, f)$ to $\left(P^{\prime}, f^{\prime}\right)$ is an isomorphism $\phi: P \rightarrow P^{\prime}$ such that $f=\phi^{*} f^{\prime} \phi$. The latter is equivalent to

$$
\tilde{f}(x, y)=\tilde{f}^{\prime}(\phi x, \phi y) \quad \forall x, y \in P \text {. }
$$

The group of isometries of $(P, f)$ is denoted by $O(f)$.

Orthogonal sums of sesquilinear forms are defined in the usual way. We denote by $\mathrm{UH}^{u}(A, \sigma)$ the category of unimodular $u$-hermitian forms over $(A, \sigma)$.

1B. Quadratic forms. Keep the setting of $1 \mathrm{~A}$. To define quadratic spaces, additional data is needed. Set

$$
\Lambda^{\min }(u)=\left\{a-a^{\sigma} u \mid a \in A\right\} \quad \text { and } \quad \Lambda^{\max }(u)=\left\{a \in A: a^{\sigma} u=-a\right\} .
$$

A form parameter (for $(A, \sigma, u)$ ) consists of an additive group $\Lambda$ such that

$$
\Lambda^{\min }(u) \subseteq \Lambda \subseteq \Lambda^{\max }(u) \quad \text { and } \quad a^{\sigma} \Lambda a \subseteq \Lambda \quad \forall a \in A .
$$

In this case, the quartet $(A, \sigma, u, \Lambda)$ is called a unitary ring. (It is also common to call the pair $(u, \Lambda)$ a form parameter.) When $2 \in A^{\times}, \Lambda^{\min }(u)=\Lambda^{\max }(u)$ because any $a \in \Lambda^{\max }(u)$ satisfies $a=\frac{1}{2} a-\left(\frac{1}{2} a\right)^{\sigma} u \in \Lambda^{\min }(u)$, so there is only one possible form parameter. 
For every $P \in \mathscr{P}(A)$, define

$$
\Lambda_{P}=\left\{f \in \operatorname{Hom}_{A}\left(P, P^{*}\right): f=-f^{*} \omega_{P} \text { and } \tilde{f}(x, x) \in \Lambda \text { for all } x \in P\right\} .
$$

Given $f \in \operatorname{Hom}_{A}\left(P, P^{*}\right)$, denote by $[f]$ the class of $f$ in $\operatorname{Hom}_{A}\left(P, P^{*}\right) / \Lambda_{P}$. A quadratic space (over $(A, \sigma, u, \Lambda)$ ) is a pair $(P,[f])$ with $P \in \mathscr{P}(A)$ and $f \in$ $\operatorname{Hom}_{A}\left(P, P^{*}\right)$. Associated with $[f]$ are the $u$-hermitian form

$$
h_{f}=f+f^{*} \omega_{P}
$$

and the quadratic map $\hat{f}: P \rightarrow A / \Lambda$ given by

$$
\hat{f}(x)=\tilde{f}(x, x)+\Lambda .
$$

Both $h_{f}$ and $\hat{f}$ are determined by the class $[f]$ (rather than $f$ ), and conversely, they also determine $[f]$. We say that $(P,[f])$ is unimodular if $h_{f}: P \rightarrow P^{*}$ is bijective.

Let $\left(P^{\prime},\left[f^{\prime}\right]\right)$ be another quadratic space. An isometry from $(P,[f])$ to $\left(P^{\prime},\left[f^{\prime}\right]\right)$ is an isomorphism $\phi: P \rightarrow P^{\prime}$ such that

$$
\left[\phi^{*} f^{\prime} \phi\right]=[f]
$$

in $\operatorname{Hom}_{A}\left(P, P^{*}\right) / \Lambda_{P}$. This is equivalent to

$$
h_{f^{\prime}}(\phi x, \phi y)=h_{f}(x, y) \quad \text { and } \quad \hat{f}^{\prime}(\phi x)=\hat{f}(x) \quad \forall x, y \in P .
$$

We let $O([f])$ denote the isometry group of $(P,[f])$.

The category of unimodular quadratic spaces over $(A, \sigma, u, \Lambda)$ is denoted by $\mathrm{UQ}^{u, \Lambda}(A, \sigma)$.

Remark 1.1. When $2 \in A^{\times},[f]$ can be recovered from $h:=h_{f}$ via $[f]=\left[\frac{1}{2} h\right]$. Equation (1.2) is therefore equivalent to $\phi^{*} h^{\prime} \phi-h \in \Lambda_{P}$ where $h^{\prime}=h_{f^{\prime}}$. Write $g=\phi^{*} h^{\prime} \phi-h$. Then $g=g^{*} \omega$ (since $h=h^{*} \omega$ and $h^{\prime}=h^{\prime *} \omega$ ) and $g=-g^{*} \omega$ (since $g \in \Lambda_{P}$ ), hence $g=0$ (because $\left.2 \in A^{\times}\right)$. Therefore, $\phi$ is an isometry from $(P,[f])$ to $\left(P^{\prime},\left[f^{\prime}\right]\right)$ if and only if it is an isometry from $(P, h)$ to $\left(P^{\prime}, h^{\prime}\right)$. It follows that $\mathrm{UQ}^{u, \Lambda}(A, \sigma) \cong \mathrm{UH}^{u}(A, \sigma)$ when $2 \in A^{\times}$.

1C. Scalar extension. Let $F$ be a commutative ring. Throughout, all tensor products are taken over $F$. A unitary $F$-algebra is a unitary ring $(A, \sigma, u, \Lambda)$ such that $A$ is an $F$-algebra, $\sigma$ is $F$-linear, and $\Lambda$ is an $F$-submodule of $A$. For a commutative ring extension $K / F$, define

$$
\mathcal{R}_{K / F}(A, \sigma, u, \Lambda)=\left(A \otimes K, \sigma \otimes \operatorname{id}_{K}, u \otimes 1_{K}, \Lambda \otimes_{F}^{A} K\right) .
$$

Here, $\Lambda \otimes_{F}^{A} K$ denotes the image of $\Lambda \otimes K$ in $A \otimes K$ (when $K$ is flat over $F$, the distinction between $\Lambda \otimes K$ and $\Lambda \otimes_{F}^{A} K$ is unnecessary). It is easy to see that $(B, \tau, v, \Gamma):=\mathcal{R}_{K / F}(A, \sigma, u, \Lambda)$ is a unitary ring.

For every $P, Q \in \mathscr{P}(A)$ and $\phi \in \operatorname{Hom}_{A}(P, Q)$, let

$$
P_{K}=P \otimes K \quad \text { and } \quad \phi_{K}=\phi \otimes \operatorname{id}_{K} \in \operatorname{Hom}_{A_{K}}\left(P_{K}, Q_{K}\right) .
$$

(Here, $P_{K}$ is considered as an $A_{K}$-module by setting $(x \otimes k)(a \otimes m)=(x a) \otimes(k m)$ for all $x \in P, a \in A, k, m \in K$.) The assignment $P \mapsto P_{K}: \mathscr{P}(A) \rightarrow \mathscr{P}\left(A_{K}\right)$ is a functor denoted by $\mathcal{R}_{K / F}$.

For every sesquilinear form $f$ on $P$, define a sesquilinear form $f_{K}$ on $P_{K}$ by linearly extending

$$
\tilde{f}_{K}\left(x \otimes k, x^{\prime} \otimes k^{\prime}\right)=\tilde{f}\left(x, x^{\prime}\right) \otimes k k^{\prime} \quad \forall x, x^{\prime} \in P, k, k^{\prime} \in K .
$$


It is easy to check that $f \in \Lambda_{P}$ implies $f_{K} \in \Gamma_{P_{K}}$, so the map sending $(P,[f])$ to $\left(P_{K},\left[f_{K}\right]\right)$ is well defined. The quadratic space (resp. sesquilinear space) $\left(P_{K},\left[f_{K}\right]\right)$ (resp. $\left.\left(P_{K}, f_{K}\right)\right)$ is called the scalar extension of $(P,[f])$ (resp. $\left.(P, f)\right)$. This gives rise to functors

$$
\begin{gathered}
\mathrm{UH}^{u}(A, \sigma) \rightarrow \mathrm{UH}^{v}(B, \tau), \\
\mathrm{UQ}^{u, \Lambda}(A, \sigma) \rightarrow \mathrm{UQ}^{v, \Gamma}(B, \tau),
\end{gathered}
$$

which, by abuse of notation, are all denoted $\mathcal{R}_{K / F}$. (The action of $\mathcal{R}_{K / F}$ on isometries is the same as its action on morphisms of $\mathscr{P}(A)$.)

We now give another description of $f_{K}$ and $\left[f_{K}\right]$, which does not pass through $\tilde{f}$ and will be useful later.

Lemma 1.2. For all $P, P^{\prime} \in \mathscr{P}(A)$, there are natural isomorphisms

$$
\operatorname{Hom}_{A}\left(P, P^{\prime}\right)_{K} \cong \operatorname{Hom}_{A_{K}}\left(P_{K}, P_{K}^{\prime}\right) \quad \text { and } \quad\left(P^{*}\right)_{K} \cong\left(P_{K}\right)^{*} \text {, }
$$

where the latter is an isomorphism of $A_{K}$-modules.

Proof. Since $P^{*}=\operatorname{Hom}_{A}(P, A)$ and $\left(P_{K}\right)^{*}=\operatorname{Hom}_{A_{K}}\left(P_{K}, A_{K}\right)$, the second isomorphism is just a special case of the first isomorphism (and it is straightforward to check that we in fact obtain an isomorphism of $A_{K}$-modules). Define $\Phi: \operatorname{Hom}_{A}\left(P, P^{\prime}\right)_{K} \rightarrow \operatorname{Hom}_{A_{K}}\left(P_{K}, P_{K}^{\prime}\right)$ by

$$
(\Phi(\phi \otimes k))(x \otimes m)=\phi x \otimes k m \quad \forall \phi \in \operatorname{Hom}_{A}\left(P, P^{\prime}\right), x \in P, k, m \in K .
$$

It is routine to verify that $\Phi$ is natural (in the categorical sense) and an isomorphism when $P=P^{\prime}=A_{A}$. The naturality of $\Phi$ now implies that it is also an isomorphism when $P$ and $P^{\prime}$ are summands of f.g. free modules.

Proposition 1.3. Every $P \in \mathscr{P}(A)$ gives rise to a commutative diagram with exact rows and such that $\alpha$ is onto and $\beta$ and $\gamma$ are isomorphisms:

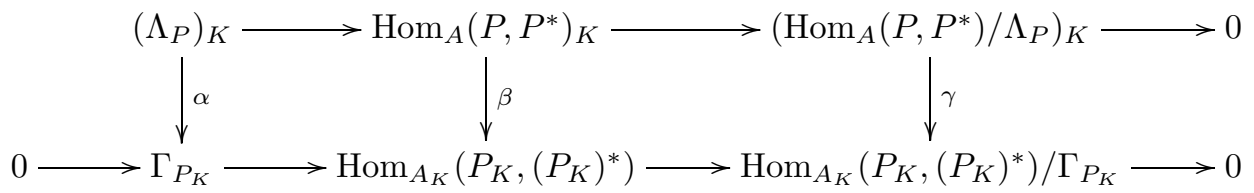

Proof. The exactness of the bottom row holds by definition, and the exactness of the top row follows from the exact sequence

$$
0 \rightarrow \Lambda_{P} \rightarrow \operatorname{Hom}_{A}\left(P, P^{*}\right) \rightarrow \operatorname{Hom}\left(P, P^{*}\right) / \Lambda_{P} \rightarrow 0
$$

by tensoring with $K$. By Lemma 1.2, we have a natural isomorphism

$$
\operatorname{Hom}_{A}\left(P, P^{*}\right)_{K} \cong \operatorname{Hom}_{A_{K}}\left(P_{K},\left(P^{*}\right)_{K}\right) \cong \operatorname{Hom}_{A_{K}}\left(P_{K},\left(P_{K}\right)^{*}\right)
$$

which we take to be $\beta$. Explicitly, for all $f \in \operatorname{Hom}_{A}\left(P, P^{*}\right)$ and $k \in K$, one has

$$
(\beta(f \otimes k))^{\tilde{}}(x \otimes a, y \otimes b)=\tilde{f}(x, y) \otimes k a b \quad \forall x, y \in P, a, b \in K .
$$

This is easily seen to imply that the image of $\left(\Lambda_{P}\right)_{K}$ in $\operatorname{Hom}_{A}\left(P, P^{*}\right)_{K}$ is mapped by $\beta$ into $\Gamma_{P_{K}}$. We define $\alpha:\left(\Lambda_{P}\right)_{K} \rightarrow \Gamma_{P_{K}}$ to be this map. The map $\gamma$ is now induced by $\alpha$ and $\beta$ in the standard way, namely, $\gamma([f] \otimes k)=[\beta(f \otimes k)]$. The five lemma implies that $\gamma$ is an isomorphism if $\alpha$ is onto, which is what we shall verify.

Assume first that $P$ is a free $A$-module with basis $\left\{x_{i}\right\}_{i=1}^{t}$. Then $\left\{y_{i}\right\}_{i}:=\left\{x_{i} \otimes 1\right\}_{i}$ is a basis of $A_{K}$. A morphism $f \in \operatorname{Hom}_{A_{K}}\left(P_{K},\left(P_{K}\right)^{*}\right)$ is completely determined by the values $\left\{\tilde{f}\left(y_{i}, y_{j}\right)\right\}_{i, j}$, and it belongs to $\Gamma_{P_{K}}$ if and only if $\tilde{f}\left(y_{i}, y_{i}\right) \in \Gamma$ and 
$\tilde{f}\left(y_{i}, y_{j}\right)+\tilde{f}\left(y_{j}, y_{i}\right)^{(\sigma \otimes \mathrm{id})}(u \otimes 1)=0$ for all $i, j$. For such $f$ and $i \leq j$, write $\tilde{f}\left(y_{i}, y_{j}\right)=\sum_{s} a_{i j}^{(s)} \otimes k_{i j}^{(s)}$ with $\left\{a_{i j}^{(s)}\right\}_{s} \subseteq A,\left\{k_{i j}^{(s)}\right\}_{s} \subseteq K$. Since $\Gamma=\Lambda \otimes_{F}^{A} K$, we can choose $a_{i i}^{(s)}$ to be in $\Lambda$ for all $i, s$. Now, for all $i \leq j$ and $s$, let $g_{i j}^{(s)}$ denote the unique morphism in $\operatorname{Hom}_{A}\left(P, P^{*}\right)$ satisfying

$$
\tilde{g}_{i j}^{(s)}\left(x_{n}, x_{m}\right)= \begin{cases}a_{i j}^{(s)}, & (n, m)=(i, j), \\ -\left(a_{i j}^{(s)}\right)^{\sigma} u, & (n, m)=(j, i), \\ 0, & \text { otherwise. }\end{cases}
$$

It is routine to verify that $g_{i j}^{(s)} \in \Lambda_{P}$ and $\alpha\left(\sum_{i \leq j} \sum_{s} g_{i j}^{(s)} \otimes k_{i j}^{(s)}\right)=f$, as required.

For general $P$, choose $P^{\prime} \in \mathscr{P}(A)$ such that $P \oplus P^{\prime}$ is free. If $f \in \Gamma_{P_{K}}$, then $f \oplus 0 \in \Gamma_{P_{K} \oplus P_{K}^{\prime}}$, hence we can write $f \oplus 0=\alpha\left(\sum_{i} g_{i} \otimes k_{i}\right)$ for $\left\{g_{i}\right\}_{i=1}^{r} \subseteq \Lambda_{P \oplus P^{\prime}}$ and $\left\{k_{i}\right\}_{i=1}^{r} \subseteq K$. For all $i$, define $h_{i} \in \operatorname{Hom}_{A}\left(P, P^{*}\right)$ by $\tilde{h}_{i}(x, y)=\tilde{g}_{i}(x \oplus 0, y \oplus 0)$. Then $\left\{h_{i}\right\}_{i=1}^{r} \subseteq \Lambda_{P}$ and an easy computation shows that $\alpha\left(\sum_{i} h_{i} \otimes k_{i}\right)=f$.

Let $P \in \mathscr{P}(A)$ and $f \in \operatorname{Hom}_{A}\left(P, P^{*}\right)$. It is straightforward to check that

$$
f_{K}=\beta\left(f \otimes 1_{K}\right) \quad \text { and } \quad\left[f_{K}\right]=\gamma\left([f] \otimes 1_{K}\right),
$$

where $\beta$ and $\gamma$ are as in Proposition 1.3 .

1D. Orthogonal unitary rings. Recall that a ring with involution $(A, \sigma)$ is simple if $A$ admits no non-trivial two-sided ideals $I$ satisfying $I^{\sigma}=I$. In this case, it is well known that $A$ is either simple, or $A \cong B \oplus B^{\text {op }}$ with $B$ a simple ring, and $\sigma$ exchanges $B$ and $B^{\mathrm{op}}$. If $A$ also happens to be artinian, then the Artin-Wedderburn Theorem implies that $A \cong \mathrm{M}_{n}(D)$ where $D$ is a division ring or a product of a division ring and its opposite.

We call a unitary ring $(A, \sigma, u, \Lambda)$ simple if $(A, \sigma)$ is simple as a ring with involution.

Proposition 1.4. Let $(A, \sigma, u, \Lambda)$ be a unitary ring such that $A$ is a semisimple ring. Then $(A, \sigma, u, \Lambda)$ factors into a product of unitary rings

$$
(A, \sigma, u, \Lambda) \cong \prod_{i=1}^{t}\left(A_{i}, \sigma_{i}, u_{i}, \Lambda_{i}\right):=\left(\prod_{i} A_{i}, \prod_{i} \sigma,\left(u_{i}\right)_{i}, \prod_{i} \Lambda_{i}\right)
$$

such that each $\left(A_{i}, \sigma_{i}, u_{i}, \Lambda_{i}\right)$ is simple artinian.

Proof. This is well known; see for instance [17, Pr. 2.7].

We now recall a notion of orthogonality for simple artinian unitary rings defined in [17, §2.4] (see also the orthogonality defined in [4, Ch. 4, §2]).

Let $A$ be a central simple algebra over a field $K$ (see for instance [25, Ch. I]). The degree and index of $A$ are denoted by $\operatorname{deg} A$ and ind $A$, respectively. Recall that involutions of the first kind on $A$ (i.e. involutions fixing $K$ pointwise) divide into two families: orthogonal and symplectic (cf. [25, §I.2]). Recall also that if char $K \neq 2$, then $\sigma$ is orthogonal if and only if $\operatorname{dim}_{K}\left\{a-a^{\sigma} \mid a \in A\right\}=\frac{1}{2} n(n-1)$ where $n=\operatorname{deg} A$.

A simple artinian unitary ring $(A, \sigma, u, \Lambda)$ is called orthogonal if:

(1) $A$ is simple as a ring and finite dimensional over its center $K$ (which is a field in this case),

(2) $\sigma$ is of the first kind (i.e. $\left.\sigma\right|_{K}=\operatorname{id}_{K}$ ), 
(3) one of the following holds:

(3a) $\operatorname{char} K \neq 2, \sigma$ is orthogonal and $u=1$,

(3b) char $K \neq 2, \sigma$ is symplectic and $u=-1$,

(3c) $\operatorname{char} K=2$ and $\Lambda=\Lambda^{\min }(u)$.

(These conditions are equivalent to $\Lambda$ being a $K$-vector space and satisfying $\operatorname{dim}_{K} \Lambda=\frac{1}{2} n(n-1)$, where $n=\operatorname{deg} A$; see [17, §2.4].)

If in addition $A$ is split (as a central simple $K$-algebra), we say that $(A, \sigma, u, \Lambda)$ is split-orthogonal.

1E. Transfer. We now recall the method of transfer into the endomorphism ring. This is a special case of transfer in hermitian categories. See [24, §II.3] or [30, Pr. 2.4] for further details.

Let $(A, \sigma, u, \Lambda)$ be a unitary ring. Fix a unimodular $u$-hermitian form $(Q, h) \in$ $\mathrm{UH}^{u}(A, \sigma)$ and let $B=\operatorname{End}_{A}(Q)$. The form $h$ induces an involution $\tau=\tau(h): B \rightarrow$ $B$ given by $\phi^{\tau}=h^{-1} \phi^{*} h$. Equivalently, $\phi^{\tau}$ is the unique element of $B$ satisfying $\tilde{h}(\phi x, y)=\tilde{h}\left(x, \phi^{\tau} y\right)$ for all $x, y \in Q$. We further define $\Gamma=\Gamma(h, \Lambda)=h^{-1} \Lambda_{Q}$. It is straightforward to check that $\left(B, \tau, 1_{B}, \Gamma\right)$ is a unitary ring. If $(P, f)$ is a sesquilinear form, we define a sesquilinear form $\left(B_{B}, \mathrm{~T}_{h} f\right)$ by

$$
\widetilde{\mathrm{T}_{h} f}\left(b, b^{\prime}\right)=b^{\tau}\left(h^{-1} f\right) b^{\prime} .
$$

It is easy to see that $f \in \Lambda_{Q}$ implies $\mathrm{T}_{h} f \in \Gamma_{B}$, hence the map $[f] \mapsto\left[\mathrm{T}_{h} f\right]$ is well defined.

Proposition 1.5 (Transfer). Keep the previous setting and identify $\operatorname{End}_{B}\left(B_{B}\right)$ with $B=\operatorname{End}_{A}(Q)$ via $\psi \mapsto \psi\left(1_{B}\right)$. Then:

(i) $[f]$ is unimodular if and only if $\left[\mathrm{T}_{h} f\right]$ is unimodular.

(ii) $O([f])=O\left(\left[\mathrm{~T}_{h} f\right]\right)$.

(iii) If $\left(P, f^{\prime}\right)$ is another sesquilinear space, then $[f] \cong\left[f^{\prime}\right]$ if and only if $\left[\mathrm{T}_{h} f\right] \cong$ $\left[\mathrm{T}_{h} f^{\prime}\right]$

More generally, there is an isomorphism between the category of quadratic spaces over $(A, \sigma, u, \Lambda)$ with base module $P$ and the category of quadratic spaces over $(B, \tau, 1, \Gamma)$ with base module $B_{B}$.

Proof. This is routine. See [24, §II.3] for a proof in a more general setting.

Remark 1.6. (i) If $(P,[f]) \in \mathrm{UQ}^{u, \Lambda}(A, \sigma)$, then by applying Proposition [1.5 with $(Q, h)=\left(P, h_{f}\right)$, we may transfer certain statements about quadratic spaces with base module $P$ to analogous statements about quadratic spaces with base module $B_{B}\left(B=\operatorname{End}_{A}(P)\right)$. This allows us to assume that the base module is isomorphic to the base ring, and free in particular.

(ii) Transfer is compatible with scalar extension in the sense of $1 \mathrm{C}$. This follows from Lemma 1.2 and left as an exercise to the reader; see also [7, §2E].

Proposition 1.7. Keep the previous setting and assume $Q \neq 0$. If $(A, \sigma, u, \Lambda)$ is simple artinian, then $(B, \tau, 1, \Gamma)$ is simple artinian. In this case, $(A, \sigma, u, \Lambda)$ is split-orthogonal if and only if $(B, \tau, 1, \Gamma)$ is split-orthogonal. 
Proof. If $A$ is simple artinian as a ring, then it is well known that $B=\operatorname{End}_{A}(Q)$ is also a simple artinian ring. If $A$ is not simple as a ring, there exists an idempotent $e \in \operatorname{Cent}(A)$ with $e^{\sigma} e=0$ and $e^{\sigma}+e=1$, and $e A e,(1-e) A(1-e)$ are simple artinian rings. This implies $Q=Q e \oplus Q(1-e)$ and $B=\operatorname{End}_{A}(Q) \cong \operatorname{End}_{e A e}(Q e) \times$ $\operatorname{End}_{(1-e) A(1-e)}(Q(1-e))$. In particular, $\operatorname{End}_{A}(Q)$ is a product of two simple artinian rings. Let $a=\operatorname{id}_{Q e} \oplus 0$ and $b=0 \oplus \operatorname{id}_{Q(1-e)}$. It is easy to check that $\tilde{h}(a x, y)=$ $\tilde{h}(x, b y)$, and hence $a^{\tau}=b$. The only non-trivial ideals of $B$ are $\operatorname{End}_{e A e}(Q e) \times 0$ and $0 \times \operatorname{End}_{(1-e) A(1-e)}(Q(1-e))$. Since these ideals contain $a$ and $b$, respectively, it follows that $B$ has no non-trivial ideals invariant under $\tau$.

Suppose now that $(A, \sigma, u, \Lambda)$ is split-orthogonal. Then $A \cong \mathrm{M}_{n}(K)$ for a field $K$, and $\sigma$ is of the first kind. Using [17, Pr. 2.5, Ex. 2.10], we may assume $\sigma$ is the transpose involution and $u=1$. Let $e$ be the matrix unit $e_{11}$. By [17, Pr. 2.4, Pr. 2.11], we may replace $(A, \sigma, u, \Lambda)$ with $\left(e A e,\left.\sigma\right|_{e A e}, e u, e \Lambda e\right)$ and $P$ with $P e$. That is, we may assume $(A, \sigma, u, \Lambda)=\left(K, \operatorname{id}_{K}, 1,0\right)$. Now, if $m=\operatorname{dim}_{K} P$, then $B \cong \mathrm{M}_{m}(K)$ and it is easy to check that $\Gamma$ is a $K$-vector space of dimension $\frac{1}{2} m(m-1)$, so $(B, \tau, 1, \Gamma)$ is split-orthogonal.

Conversely, assume that $(B, \tau, 1, \Gamma)$ is split-orthogonal. If $A$ is not simple artinian, then the argument above implies that the involution on $B$ is of the second kind, which is impossible. Thus, $A$ is simple artinian. In fact, if $B \cong \mathrm{M}_{m}(K)$ where $K$ is a field, then $A \cong \mathrm{M}_{n}(K)$ for some $n \in \mathbb{N}$. Identifying $\operatorname{Cent}(A)$ and $\operatorname{Cent}(B)$ with $K$, it is easy to see that $\left.\sigma\right|_{K}=\left.\tau\right|_{K}$, hence $\sigma$ is of the first kind. As in the previous paragraph, we may again assume $A=K$ and $\sigma=\mathrm{id}_{K}$. This implies $u \in\{ \pm 1\}$. If char $K \neq 2$ and $u=-1$, then $h$ is an alternating $K$-bilinear form, and so $\tau$ is symplectic, which is impossible. Thus, $u=1$. Now, take an $A$-basis $\left\{x_{1}, \ldots, x_{m}\right\}$ of $P$ with dual basis $\left\{\phi_{1}, \ldots, \phi_{m}\right\} \subseteq P^{*}$, and let $f_{i j}=\left[x \mapsto x_{i}\left(\phi_{j} x\right)\right] \in \operatorname{Hom}_{A}\left(P, P^{*}\right)$. Then $\left\{f_{i j}\right\}_{1 \leq i, j \leq m}$ is a $K$-basis of $\operatorname{Hom}_{A}\left(P, P^{*}\right)$ and it is easy to check that $\Lambda_{P}=\sum_{i<j}\left(f_{i j}-f_{j i}\right) K+\sum_{i} f_{i i} \Lambda$. Thus, $\Gamma=\sum_{i<j} h^{-1}\left(f_{i j}-f_{j i}\right) K+\sum_{i} h^{-1} f_{i i} \Lambda$, while $\Gamma^{\min }(1)=\sum_{i<j} h^{-1}\left(f_{i j}-f_{j i}\right) K$ (note that $\left(h^{-1} f_{i j}\right)^{\tau}=h^{-1} f_{j i}$; use the identities $h=h^{*} \omega_{P}$ and $\left.f_{i j}^{*} \omega_{P}=f_{j i}\right)$. Since $(B, \tau, 1, \Gamma)$ is split-orthogonal, we have $\Gamma=\Gamma^{\min }(1)$, so $\Lambda=0$, as required.

1F. The Dickson map. Let $(A, \sigma, u, \Lambda)$ be a split-orthogonal simple artinian unitary ring, let $K=\operatorname{Cent}(A)$ and let $(P,[f]) \in \mathrm{UQ}^{u, \Lambda}(A, \sigma)$. The Dickson map (also called pseudodeterminant or quasideterminant) is a surjective group homomorphism

$$
\Delta=\Delta_{[f]}: O([f]) \rightarrow \mathbb{Z} / 2 \mathbb{Z} .
$$

In case $2 \in A^{\times}$, it can be defined using the reduced norm in $E:=\operatorname{End}_{A}(P)$ via

$$
\operatorname{Nrd}_{E / K}(\psi)=(-1)^{\Delta(\psi)} \quad \forall \psi \in O([f])
$$

and, in general, it can be defined by

$$
\Delta(\psi)=\frac{\operatorname{dim}_{K}(1-\psi) E}{\operatorname{deg} E}+2 \mathbb{Z} \quad \forall \psi \in O([f]) .
$$

(This indeed yields a group homomorphism.) Viewing $O([f])$ as an affine group scheme over $K$ (see the appendix) and $\mathbb{Z} / 2 \mathbb{Z}$ as a constant group scheme over $K$, $\Delta$ becomes a morphism of algebraic groups (defined over $K$ ), and the kernel of $\Delta$ is the (Zariski) connected component of $O([f])$. See [17, §5.1] and the references therein for proofs and further discussion. 


\section{Some topology}

In this section, we recall several facts allowing us to properly topologize various algebraic objects.

Let $K$ be a commutative ring and let Comm- $K$ denote the category of commutative $K$-algebras. By a scheme over $K$ or a $K$-scheme we mean a scheme

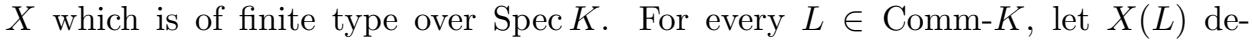
note the $L$-points of $X$ (i.e. the set of $K$-morphisms Spec $L \rightarrow X$ ). The map $X \mapsto X(L):$ Comm- $K \rightarrow$ Set is a functor called the functor of points of $X$. This functor is representable precisely when $X$ is an affine scheme (over $K$ ). In this case, Yoneda's Lemma implies that the functor $L \mapsto X(L)$ determines $X$ up to isomorphism; see for instance [38, §2].

Proposition 2.1. Let $K$ be a Hausdorff topological commutative ring. There is a unique way to topologize the set of $K$-points of all affine $K$-schemes $X$ such that:

(1) The assignment $X \mapsto X(K)$ is a functor from affine $K$-schemes to topological spaces, and it is compatible with fibered products.

(2) Closed immersions become closed embeddings when restricted to $K$-points.

(3) $\mathbb{A}_{K}^{1}(K)=K$ is given the topology of $K$.

Proof. See [14, Pr. 2.1]. Explicitly, if $X=\operatorname{Spec} A$ for a commutative $K$-algebra $A$, then the topology on $X(K) \cong \operatorname{Hom}_{\mathrm{Comm}-K}(A, K)$ is the subspace topology induced from the product topology on $\operatorname{Hom}_{\text {Set }}(A, K)=K^{A}$.

Example 2.2. It follows from the proposition that if $X$ is an affine scheme over $K$ and $X \rightarrow \mathbb{A}_{K}^{n}$ is a closed immersion, then the topology on $X(K)$ is induced from the product topology on $\mathbb{A}_{K}^{n}(K)=K^{n}$. The resulting topology is independent of the immersion $X \rightarrow \mathbb{A}_{K}^{n}$.

The following proposition allows us to realize finitely generated projective modules over $K$ as the $K$-points of affine schemes. Recall that $\mathscr{P}(K)$ denotes the category of finitely generated projective right $K$-modules.

Proposition 2.3. There is a functor $P \mapsto \underline{P}$ from $\mathscr{P}(K)$ to the category of affine schemes over $K$ such that:

(1) The functors $\left[L \mapsto P_{L}:=P \otimes_{K} L\right]$ and $[L \mapsto \underline{P}(L)]$ from Comm-K to Set are isomorphic.

(2) If $Q$ is a summand of $P$, then the corresponding morphism $\underline{Q} \rightarrow \underline{P}$ is a closed immersion.

(3) $P \oplus Q$ is canonically isomorphic to $\underline{P} \times \operatorname{Spec} K \underline{Q}$.

(4) $\overline{K^{n}}$ is isomorphic to $\mathbb{A}_{K}^{n}$.

Proof. This is well known: Let $P^{\vee}:=\operatorname{Hom}_{K}(P, K)$, let $S^{n} P^{\vee}$ be the $n$-th symmetric (tensor) power of $P^{\vee}$, and let $S P^{\vee}=\bigoplus_{n=0}^{\infty} S^{n} P^{\vee}$ be the free symmetric algebra spanned by $P^{\vee}$. Then $\underline{P}:=\operatorname{Spec} S P^{\vee}$ fulfills all the requirements. If $\phi: Q \rightarrow P$ is a $K$-linear homomorphism, then the corresponding morphism $Q \rightarrow \underline{P}$ comes from the induced map $\bigoplus_{n \geq 0} S^{n} \phi^{\vee}: S P^{\vee} \rightarrow S Q^{\vee}$. The details are left as an exercise to the reader; see [25, Ex. 20.2(2)] for the case where $K$ is a field.

Suppose henceforth that $K$ is a Hausdorff topological commutative ring such that $K^{\times}$is open in $K$ and the inversion map $k \mapsto k^{-1}: K^{\times} \rightarrow K^{\times}$is continuous. By Proposition 2.3, we can realize all finitely generated projective $K$-modules as the 
$K$-points of affine schemes over $K$, and topologize them using Proposition 2.1. For every $P \in \mathscr{P}(K)$, denote by $\tau_{P}$ the topology obtained in this way. It is immediate to check that this topology has the following properties:

(1) All $K$-linear homomorphisms are continuous.

(2) For all $P, Q \in \mathscr{P}(K)$, the topology $\tau_{P \times Q}$ coincides with the product topology on $P \times Q$.

(3) $\tau_{K}$ is the topology of $K$ as a ring.

As a result, if $P \in \mathscr{P}(K)$ is isomorphic to a summand of $K^{n}$, then $\tau_{P}$ coincides with the subspace topology induced from the inclusion $P \rightarrow K^{n}$.

Proposition 2.4. Let $P_{1}, \ldots, P_{t}, Q \in \mathscr{P}(K)$. Then any $K$-multilinear map $\mu$ : $P_{1} \times \cdots \times P_{t} \rightarrow Q$ is continuous.

Proof. It is enough to show that $\mu$ is induced by a $K$-morphism $\underline{\mu}: \underline{P_{1}} \times \cdots \times \underline{P_{t}} \rightarrow \underline{Q}$. By Yoneda's Lemma, this holds if $\mu$ extends to a natural transformation from the points functor of $P_{1} \times \cdots \times \underline{P}_{t}$ to the points functor of $Q$. Namely, for all $L \in$ Comm- $K$, there is a map $\mu_{L}:\left(P_{1}\right)_{L} \times \cdots \times\left(P_{t}\right)_{L} \rightarrow Q_{L}$ such that $\mu_{K}=\mu$ and $\left\{\mu_{L}\right\}_{L \in \text { Comm- } K}$ is a natural transformation from $\left[L \mapsto\left(P_{1} \times \cdots \times \underline{P}_{t}\right)(L)=\right.$ $\left.\left(P_{1}\right)_{L} \times \cdots \times\left(P_{t}\right)_{L}\right]$ to $\left[L \mapsto Q(L)=Q_{L}\right]$. Indeed, it is easy to check that the map $\mu_{L}\left(p_{1} \otimes \ell_{1}, \ldots, p_{t} \otimes \ell_{t}\right)=\mu\left(p_{1}, \ldots, p_{t}\right) \otimes \prod_{i} \ell_{i}\left(\right.$ with $p_{1} \in P_{1}, \ldots, p_{t} \in P_{t}$, $\left.\ell_{1}, \ldots, \ell_{t} \in L\right)$ fulfills these requirements.

Proposition 2.5. Every $P \in \mathscr{P}(K)$ is a topological $K$-module.

Proof. The addition and subtraction maps from $P \times P$ to $P$ are continuous because they are $K$-linear. The map $(p, k) \mapsto p k: P \times K \rightarrow P$ is continuous because it is $K$-multilinear (Proposition 2.4).

Proposition 2.6. Let $A$ be a $K$-algebra such that $A \in \mathscr{P}(K)$. Then:

(i) $A$ is a topological $K$-algebra (when topologized as an f.g. projective $K$ module).

(ii) $A^{\times}$is open in $A$ and the map $a \mapsto a^{-1}: A^{\times} \rightarrow A^{\times}$is continuous.

Proof. (i) The algebra $A$ is a topological $K$-module by Proposition 2.5 and the multiplication in $A$ is continuous by Proposition 2.4.

(ii) If $A \cong \mathrm{M}_{n}(K)$ as $K$-algebras, then this follows from the fact that the determinant map is continuous, and the assumptions that $K^{\times}$is open in $K$ and the map $a \mapsto a^{-1}: K^{\times} \rightarrow K^{\times}$is continuous.

For general $A$, choose $P \in \mathscr{P}(K)$ such that $A \oplus P$ is free and let $E=\operatorname{End}_{K}(A \oplus P)$ and $B=\operatorname{End}_{K}(A)$. Then $E \cong \mathrm{M}_{n}(K)$ for some $n$, and hence $E^{\times}$is open in $E$ and the inversion map $i: E^{\times} \rightarrow E^{\times}$is continuous. Define $f: B \rightarrow E$ by $f(\phi)=\left(\phi \oplus 0_{P}\right)+\left(0_{A} \oplus \operatorname{id}_{P}\right)$ and $g: E \rightarrow B$ by letting $g(\phi)$ be the unique element of $B$ satisfying $g(\phi) \oplus 0_{P}=\left(\mathrm{id}_{A} \oplus 0_{P}\right) \phi\left(\operatorname{id}_{A} \oplus 0_{P}\right)$. Then $f$ and $g$ are continuous. Since $B^{\times}=f^{-1}\left(E^{\times}\right), B^{\times}$is open in $B$, and since $\left.g \circ i \circ f\right|_{B^{\times}}$is the inversion map of $B$, the map $b \mapsto b^{-1}: B^{\times} \rightarrow B^{\times}$is continuous.

Now define $t: A \rightarrow B=\operatorname{End}_{K}(A)$ by $t(a)=[x \mapsto a x]$ and $s: B \rightarrow A$ by $s(b)=b\left(1_{A}\right)$. It is easy to check that $t$ is a homomorphism of $K$-algebras whose image is $\operatorname{End}_{A}\left(A_{A}\right)$, and $s \circ t=\mathrm{id}_{A}$. Thus, $A$ is a summand of $B$ via $t$, and hence $t$ is a closed embedding. Since $\operatorname{End}_{A}\left(A_{A}\right) \cap B^{\times}=\operatorname{End}_{A}\left(A_{A}\right)^{\times}$, we have $t^{-1}\left(B^{\times}\right)=A^{\times}$. It follows that $A^{\times}$is open in $A$ and $a \mapsto a^{-1}: A^{\times} \rightarrow A^{\times}$is continuous. 
Proposition 2.7. Let $P \in \mathscr{P}(K)$. Then the topology of $\operatorname{End}_{K}(P)$ coincides with the topology induced from the product topology on $P^{P}=\operatorname{End}_{\text {Set }}(P)$.

Proof. Recall that a (finite) dual basis for a module $P$ consists of a finite collection $\left\{p_{i}, \psi_{i}\right\}_{i \in I}$ such that $p_{i} \in P, \psi_{i} \in P^{\vee}$ and $x=\sum_{i} p_{i}\left(\psi_{i} x\right)$ for all $x \in P$. A module is finitely generated projective if and only if it admits a dual basis (26, Lm. 2.9, Rm. 2.11]).

Let $\left\{p_{i}, \psi_{i}\right\}_{i=1}^{n}$ be a dual basis for $P$ and write $E=\operatorname{End}_{K}(P)$. Define $\Phi: E \rightarrow P^{n}$ and $\Psi: P^{n} \rightarrow E$ by $\Phi f=\left(f p_{1}, \ldots, f p_{n}\right)$ and $\left(\Psi\left(x_{1}, \ldots, x_{n}\right)\right) x=\sum_{i} x_{i}\left(\psi_{i} x\right)$ (for all $\left.x, x_{1}, \ldots, x_{n} \in P\right)$. It is straightforward to check that $\Psi \Phi=\mathrm{id}$, hence $E$ is a summand of $P^{n}$. Thus, $\tau_{E}$ is induced from $\tau_{P^{n}}$ via the embedding $\Phi$.

Let $\tau$ denote the topology induced on $E$ from the embedding $E \rightarrow P^{P}$ (where $P^{P}$ is given the product topology). Since $\Phi$ factors through the embedding $E \rightarrow P^{P}$, and the factor map $P^{P} \rightarrow P^{n}$ is continuous, we have $\tau_{E} \subseteq \tau$. To see the converse, let $U$ be a $\tau$-neighborhood of some $f \in E$. Then there exists a neighborhood $V$ of $f$ in $P^{P}$ whose inverse image in $E$ is $U$. By the definition of the product topology, there exist $p_{n+1}, \ldots, p_{m} \in P$ and open sets $\left\{U_{i}\right\}_{i=n+1}^{m} \subseteq \tau_{P}$ such that $f p_{i} \in U_{i}$ for all $n<i \leq m$, and $V \supseteq \prod_{i=n+1}^{m} U_{i} \times P^{P \backslash\left\{p_{n+1}, \ldots, p_{m}\right\}}$. Define $\psi_{i}=0 \in P^{\vee}$ for all $n<i \leq m$. Then $\left\{p_{i}, \psi_{i}\right\}_{i=1}^{m}$ is also a dual basis of $P$. Replacing $\left\{p_{i}, \psi_{i}\right\}_{i=1}^{n}$ (which is arbitrary) with $\left\{p_{i}, \psi_{i}\right\}_{i=1}^{m}$, we see that

$$
f \in \Phi^{-1}\left(\prod_{1 \leq i \leq n} P \times \prod_{n<i \leq m} U_{i}\right) \subseteq U .
$$

It follows that every $\tau$-neighborhood of $f$ contains a $\tau_{E}$-neighborhood of $f$, so $\tau \subseteq \tau_{E}$.

Suppose now that $F$ is a subring of $K$. For any $Q \in \mathscr{P}(F)$, let $Q_{K}:=Q \otimes_{F} K \in$ $\mathscr{P}(K)$. Similar notation will be applied to $F$-algebras and $F$-homomorphisms. We view $Q$ as an $F$-submodule of $Q_{K}$ by identifying $x \in Q$ with $x \otimes 1_{K}$ (the map $x \mapsto x \otimes 1_{K}$ is injective for free modules, and hence for all projective modules).

Proposition 2.8. Let $Q \in \mathscr{P}(F)$. If $F$ is dense in $K$, then $Q$ is dense in $Q_{K}$.

Proof. Let $U$ be an open subset of $Q_{K}$ and let $x \in U$. Then $x=\sum_{i} x_{i} b_{i}$ for some $b_{1}, \ldots, b_{n} \in K$ and $x_{1}, \ldots, x_{n} \in Q$. Since $Q_{K}$ is a topological $K$-module, there are neighborhoods $b_{i} \in U_{i} \in \tau_{K}(1 \leq i \leq n)$ such that $\sum_{i} x_{i} U_{i} \subseteq U$. Since $F$ is dense in $K$, there are $a_{i} \in U_{i} \cap F(1 \leq i \leq n)$. Then $\sum_{i} x_{i} a_{i} \in U \cap Q$.

Proposition 2.9. Assume that $F^{\times}=F \cap K^{\times}$and let $A$ be an $F$-algebra such that $A \in \mathscr{P}(F)$. Then $A^{\times}=A \cap A_{K}^{\times}$. Furthermore, if $F$ is dense in $K$, then $A^{\times}$is dense in $A_{K}^{\times}$.

Proof. It is clear that $A^{\times} \subseteq A \cap A_{K}^{\times}$. To see the converse, let $a \in A \cap A_{K}^{\times}$and let $Q \in \mathscr{P}(F)$ be such that $A \oplus Q \cong F^{n}$. Define $t: A \rightarrow \operatorname{End}_{F}(A)$ as in the proof of Proposition 2.6. Then $a$ is invertible if and only if $t(a) \oplus \operatorname{id}_{Q} \in \operatorname{End}_{F}(A \oplus Q) \cong$ $\mathrm{M}_{n}(F)$ is invertible, which is equivalent to $\operatorname{det}\left(t(a) \oplus \operatorname{id}_{Q}\right) \in F^{\times}$. Since $t(a)_{K}$ : $A_{K} \rightarrow A_{K}$ is invertible, then so is $t(a)_{K} \oplus \operatorname{id}_{Q_{K}} \in \operatorname{End}_{K}\left(A_{K} \oplus Q_{K}\right) \cong \mathrm{M}_{n}(K)$, hence $\operatorname{det}\left(t(a)_{K} \oplus \operatorname{id}_{Q_{K}}\right) \in K^{\times}$. On the other hand $\operatorname{det}\left(t(a)_{K} \oplus \operatorname{id}_{Q_{K}}\right)=\operatorname{det}\left(t(a) \oplus \operatorname{id}_{Q}\right) \in$ $F$, and so $\operatorname{det}\left(t(a) \oplus \operatorname{id}_{Q}\right) \in F \cap K^{\times}=F^{\times}$(by assumption). Thus, $t(a) \oplus \operatorname{id}_{Q}$ is invertible, as required.

To finish, if $F$ is dense in $K$, then by Proposition [2.8, $A$ is dense in $A_{K}$. By Proposition 2.6. $A_{K}^{\times}$is open $A_{K}$, so $A^{\times}=A \cap A_{K}^{\times}$is dense in $A_{K}^{\times}$. 
Remark 2.10. A subring $F \subseteq K$ satisfying $F^{\times}=F \cap K^{\times}$is called rationally closed (in $K$ ). If $F$ is artinian, then it automatically satisfies this condition, because being a non-unit in $F$ is equivalent to being a zero divisor.

Let $(A, \sigma, u, \Lambda)$ be a unitary $K$-algebra and let $(P,[f])$ be a unimodular quadratic space over $(A, \sigma, u, \Lambda)$. When the functor $L \mapsto O\left(\left[f_{L}\right]\right):$ Comm- $K \rightarrow$ Grp is representable, there exists an affine group scheme over $K$, denoted $\mathbf{O}([f])$, such that the functors $\left[L \mapsto O\left(\left[f_{L}\right]\right)\right]$ and $[L \mapsto \mathbf{O}([f])(L)]$ are isomorphic. In this case, we say that $\mathbf{O}([f])$ is defined as an affine $K$-scheme.

When $A \in \mathscr{P}(K)$ and $\Lambda$ is a summand of $A$, it is always true that $\mathbf{O}([f])$ is defined as an affine $K$-scheme. Furthermore, in this case, $\operatorname{End}_{A}(P)$ is projective (being a $K$-summand of $\operatorname{End}_{A}\left(A^{n}, A^{m}\right) \cong A^{n m}$ for some $\left.n, m \in \mathbb{N}\right)$ and the inclusion map $O([f]) \rightarrow \operatorname{End}_{A}(P)$ is induced from a closed embedding $\mathbf{O}([f]) \rightarrow \operatorname{End}_{A}(P)$. These somewhat technical facts are verified in the appendix.

\section{WEAK APPROXIMATION}

Let $K$ be a commutative semilocal topological ring and let $F$ be a dense subring of $K$. Let $(A, \sigma, u, \Lambda)$ be a unitary $F$-algebra and let $(P,[f]) \in \mathrm{UQ}^{u, \Lambda}(A, \sigma)$. In this section, we prove a weak approximation theorem for the group $O([f])$. Namely, we will show that under mild assumptions, the closure of the image of $O([f])$ in $O\left(\left[f_{K}\right]\right)$ has finite index (which can be bounded effectively). This result will play an important role in the following sections.

To give the flavor of the proof, let us sketch an ad-hoc proof in case $F$ and $K$ are fields of characteristic not 2 and $(A, \sigma, u, \Lambda)=\left(F, \mathrm{id}_{F}, 1,0\right)$ : In this case, the Cartan-Dieudonné Theorem implies that $O\left(\left[f_{K}\right]\right)$ is a generated by reflections. Every reflection of $\left[f_{K}\right]$ can be topologically approximated by a reflection of $[f]$, and hence $O([f])$ is dense in $O\left(\left[f_{K}\right]\right)$. The proof that we give here follows essentially the same lines; the Cartan-Dieudonné Theorem will be replaced with a certain generalization to semilocal base rings (see the introduction of [17] for a brief survey of such results).

We note that weak approximation theorems for reductive algebraic groups over general topological fields were studied by many authors, especially in the context of adjoint groups. See [36] and references therein for positive and negative results results. In fact, it is possible that our approximation result (Theorem 3.4 Corollary 3.7) can be deduced from such known results, at least when $F$ is a field and $A$ is a separable $F$-algebra. The methods we use here have the advantage of avoiding reductiveness issues, not using any valuation theory (any topological field works), and generalizing to semilocal rings.

$3 \mathrm{~A}$. Generation by pseudo-reflections. Let $(A, \sigma, u, \Lambda)$ be a unitary ring and let $(P,[f]) \in \mathrm{UQ}^{u, \Lambda}(A, \sigma)$. For every $y \in P$ and $c \in \hat{f}(y) \cap A^{\times}$, we define $s_{y, c}: P \rightarrow$ $P$ by

$$
s_{y, c}(x)=x-y \cdot c^{-1} \cdot \tilde{h}_{f}(y, x) \quad \forall x \in P .
$$

Following [12, IV.§2], we call $s_{y, c}$ a pseudo-reflection of $[f]$, or just a reflection for short. It is well-known that $s_{y, c} \in O([f])$ and $s_{y, c}^{-1}=s_{y, c^{\sigma} u}$; see [32, §1] or [17, §3]. Denote by $O^{\prime}([f])$ the subgroup of $O([f])$ generated by reflections.

We now recall a theorem from [17] describing the group $O^{\prime}([f])$ in case $A$ is semilocal and $P$ is free; see also 32 for conditions guaranteeing that $O^{\prime}([f])=$ $O([f])$. Recall that $A$ is semilocal if $A / \operatorname{Jac}(A)$ is semisimple artinian $(\operatorname{Jac}(A)$ 
denotes the Jacobson radical). If in addition idempotents lift modulo $\operatorname{Jac}(A)$, then $A$ is called semiperfect. For example, all one-sided artinian rings are semiperfect; see [33, §2.7] for further examples and details.

Assume $A$ is semilocal. We set some general notation: Let $\bar{A}:=A / \operatorname{Jac}(A)$, let $\bar{\sigma}$ be the involution induced by $\sigma$ on $\bar{A}$, and set $\bar{a}=a+\operatorname{Jac}(A)$ for all $a \in A$. Then $(\bar{A}, \bar{\sigma}, \bar{u}, \bar{\Lambda})$ is a semisimple unitary ring, hence by Proposition 1.4 , it factors into a product

$$
(\bar{A}, \bar{\sigma}, \bar{u}, \bar{\Lambda})=\prod_{i=1}^{t}\left(A_{i}, \sigma_{i}, u_{i}, \Lambda_{i}\right)
$$

where the factors are simple artinian unitary rings (see 1D). We further write $A_{i}=\mathrm{M}_{n_{i}}\left(D_{i}\right)$ where $D_{i}$ is a division ring or a product of a division ring and its opposite.

Every quadratic space $(P,[f])$ over $(A, \sigma, u, \Lambda)$ gives rise to a quadratic space $\underline{(\bar{P},[\bar{f}])}$ over $(\bar{A}, \bar{\sigma}, \bar{u}, \bar{\Lambda})$. Namely, $\bar{P}=P / P \operatorname{Jac}(A)$ and $\tilde{\bar{f}}$ is given by $\tilde{\tilde{f}}(\bar{x}, \bar{y})=$ $\overline{\tilde{f}(x, y)}$ for all $x, y \in P$ (where $\bar{x}=x+P \operatorname{Jac}(A)$ ). This in turn gives rise to quadratic spaces $\left(P_{i},\left[f_{i}\right]\right)_{i=1}^{t}$ over $\left(A_{i}, \sigma_{i}, u_{i}, \Lambda_{i}\right)_{i=1}^{t}$; if one writes $\bar{P}=\prod_{i} P_{i}$ with $P_{i} \in \mathscr{P}\left(A_{i}\right)$, then $\tilde{f}_{i}$ is just the restriction of $\tilde{\bar{f}}$ to $P_{i} \times P_{i}$. It is well-known that if $(P,[f])$ is unimodular, then so are $\left(P,\left[f_{i}\right]\right)_{i=1}^{t}$ (see [17, Lm. 4.3(ii)] and more generally [24, §I.7.1]).

Every isometry $\phi \in O([f])$ induces an isometry $\bar{\phi} \in O([\bar{f}])$ given by $\bar{\phi}(\bar{x})=$ $\overline{\phi x}$. Restricting $\bar{\phi}$ to $P_{i}$ yields an isometry $\phi_{i} \in O\left(\left[f_{i}\right]\right)$. It is easy to check that $\phi \mapsto \phi_{i}: O([f]) \rightarrow O\left(\left[f_{i}\right]\right)$ is a group homomorphism. When $\left(A_{i}, \sigma_{i}, u_{i}, \Lambda_{i}\right)$ is split-orthogonal (see 1D) and $(P,[f])$ is unimodular, we define

$$
\begin{aligned}
\Delta_{i}=\Delta_{i,[f]}: \quad O([f]) & \rightarrow \mathbb{Z} / 2 \mathbb{Z} \\
\phi & \mapsto \Delta_{\left[f_{i}\right]}\left(\phi_{i}\right),
\end{aligned}
$$

where $\Delta_{\left[f_{i}\right]}$ is the Dickson map of $\left[f_{i}\right]$ (see $1 \mathrm{~F}$ ). If $\mathcal{I}$ is any subset of $\{1, \ldots, t\}$ consisting of indices $i$ for which $\left(A_{i}, \sigma_{i}, u_{i}, \Lambda_{i}\right)$ is split-orthogonal, we define

$$
\Delta_{\mathcal{I}}=\Delta_{\mathcal{I},[f]}: O([f]) \rightarrow(\mathbb{Z} / 2 \mathbb{Z})^{\mathcal{I}}
$$

by $\Delta_{\mathcal{I}}(\phi)=\left(\Delta_{i}(\phi)\right)_{i \in \mathcal{I}}$.

Theorem 3.1. Let $(P,[f]) \in \mathrm{UQ}^{u, \Lambda}(\sigma, A)$. Assume that $P$ is free and for all $1 \leq i \leq t, D_{i}$ is not isomorphic to $\mathbb{F}_{2}$ or $\mathbb{F}_{2} \times \mathbb{F}_{2}$. Let $\mathcal{I}=\mathcal{I}(A)$ denote the set of $i$ 's for which $\left(A_{i}, \sigma_{i}, u_{i}, \Lambda_{i}\right)$ is split-orthogonal, and let $\xi=\left(n_{i}+2 \mathbb{Z}\right)_{i \in \mathcal{I}}=$ $\left(\operatorname{deg} A_{i}+2 \mathbb{Z}\right)_{i \in \mathcal{I}} \in(\mathbb{Z} / 2 \mathbb{Z})^{\mathcal{I}}$. Then

$$
O^{\prime}([f])=\Delta_{\mathcal{I}}^{-1}(\{0, \xi\}) .
$$

When $A$ is semiperfect, the theorem holds under the milder assumption that $P_{i} \neq 0$ for all $1 \leq i \leq t$ ( $P$ does not have to be free). Furthermore, in this case, $\Delta_{\mathcal{I}}$ is onto.

Proof. See [17, Th. 5.8, Th. 5.10].

Corollary 3.2 ([17, Cr. 5.9]). In the setting of Theorem $\left[3.1,\left[O([f]): O^{\prime}([f])\right]\right.$ is a finite power of 2 . 
3B. The Dickson map and transfer. Keep the setting of 3A We now verify that the map $\Delta_{\mathcal{I}}$ of Theorem 3.1 is compatible with transfer in the sense of $1 \mathrm{E}$

Let $(P,[f]) \in \mathrm{UQ}^{u, \Lambda}(A, \sigma)$ and define $(B, \tau, 1, \Gamma)$ as in $1 \mathrm{E}$ with $(Q, h)=\left(P, h_{f}\right)$. Write also $[g]=\left[\mathrm{T}_{h} f\right]$ and identify $O([f])$ with $O([g])$ as in Proposition 1.5. Similarly to $\left[3 \mathrm{~A}\right.$, we write $(\bar{B}, \bar{\tau}, \overline{1}, \bar{\Gamma})=\prod_{j=1}^{s}\left(B_{j}, \tau_{j}, 1, \Gamma_{j}\right)$ where the factors are simple artinian unitary rings. This gives rise to quadratic spaces $\left(B_{i},\left[g_{i}\right]\right) \in \mathrm{UQ}^{1, \Gamma_{i}}\left(B_{i}, \tau_{i}\right)$. Finally, let $\mathcal{I}(B)$ be the set of $j$ 's for which $\left(B_{j}, \tau_{j}, 1, \Gamma_{j}\right)$ is split-orthogonal and let $\mathcal{I}(P)$ be the set of $i$ 's for which $\left(A_{i}, \sigma_{i}, u_{i}, \Lambda_{i}\right)$ is split orthogonal and $P_{i} \neq 0$.

Proposition 3.3. There is an isomorphism $\mathcal{I}(B) \cong \mathcal{I}(P)$ such that the induced isomorphism $(\mathbb{Z} / 2 \mathbb{Z})^{\mathcal{I}(B)} \cong(\mathbb{Z} / 2 \mathbb{Z})^{\mathcal{I}(P)}$ makes the following diagram commute:

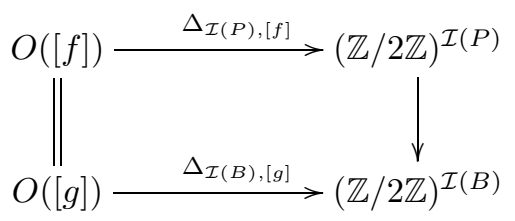

Proof. We first claim that $\operatorname{Jac}(B)=\operatorname{Hom}_{A}(P, P \operatorname{Jac}(A))$ and $B / \operatorname{Jac}(B) \cong \operatorname{End}_{\bar{A}}(\bar{P})$. This is a standard argument: It is easy to see that for all $P, Q \in \mathscr{P}(A)$, we have a natural isomorphism $\operatorname{Hom}_{A}(P, Q) / \operatorname{Hom}_{A}(P, Q \operatorname{Jac}(A)) \cong \operatorname{Hom}_{\bar{A}}(\bar{P}, \bar{Q})$ (check this when $P=Q=A_{A}$ and then extend to general $P$ and $Q$ using the naturality). Thus, $B / \operatorname{Hom}_{A}(P, P \operatorname{Jac}(A)) \cong \operatorname{End}_{\bar{A}}(\bar{P})$ (as rings). Since $\bar{A}$ is semisimple, $B / \operatorname{Hom}_{A}(P, P \operatorname{Jac}(A))$ is semisimple and hence $\operatorname{Jac}(B) \subseteq \operatorname{Hom}_{A}(P, P \operatorname{Jac}(A))$. To see the other inclusion, observe that if $\phi \in 1+\operatorname{Hom}_{A}(P, P \operatorname{Jac}(A))$, then $\operatorname{im}(\phi)+P \operatorname{Jac}(A)=P$, so by Nakayama's Lemma, $\phi$ is onto. Since $P$ is projective, $\phi$ admits a right inverse. It follows that $1+\operatorname{Hom}_{A}(P, P \operatorname{Jac}(A))$ consists of right-invertible elements, hence $\operatorname{Hom}_{A}(P, P \operatorname{Jac}(A)) \subseteq \operatorname{Jac}(B)$.

Since $\bar{A}=\prod_{i} A_{i}$, we have $\bar{B} \cong \operatorname{End}_{\bar{A}}(\bar{P})=\prod_{i=1}^{t} \operatorname{End}_{A_{i}}\left(P_{i}\right)$ (it is possible that $\left.P_{i}=0\right)$. By 1E the hermitian space $\left(P_{i}, h_{f_{i}}\right)$ induces a unitary ring structure on $\operatorname{End}_{A_{i}}\left(P_{i}\right)$. The resulting unitary ring structure on $\prod_{i=1}^{t} \operatorname{End}_{A_{i}}\left(P_{i}\right)$ is easily seen to coincide with the one on $\bar{B}$. We may therefore identify $\bar{B}$ and $\prod_{i=1}^{t} \operatorname{End}_{A_{i}}\left(P_{i}\right)$ as unitary rings. Let $\mathcal{J}=\left\{1 \leq i \leq t \mid P_{i} \neq 0\right\}$. By Proposition [1.7 the rings $\operatorname{End}_{A_{i}}\left(P_{i}\right)$ are simple artinian as unitary rings, and $\operatorname{End}_{A_{i}}\left(P_{i}\right)$ is split-orthogonal if and only $A_{i}$ is split-orthogonal and $P_{i} \neq 0$. This gives rise to a bijection $\alpha$ : $\{1, \ldots, s\} \rightarrow \mathcal{J}$ such that for all $j, B_{j} \cong \operatorname{End}_{A_{\alpha(j)}}\left(P_{\alpha(j)}\right)$ as unitary rings, and $B_{j}$ is split-orthogonal if and only if $A_{\alpha(j)}$ is split-orthogonal. In particular, $\alpha$ restricts to a bijection between $\mathcal{I}(B)$ and $\mathcal{I}(P)$.

The commutativity of the diagram follows directly from the definition of the maps $\Delta_{\alpha(j),[f]}$ and $\Delta_{j,[g]}(j \in \mathcal{I}(B))$ (see 1F); it depends only on the isomorphism class of the ring $\operatorname{End}_{A_{\alpha(j)}}\left(P_{\alpha(j)}\right) \cong B_{j} \cong \operatorname{End}_{B_{j}}\left(B_{j}\right)$.

3C. Weak approximation. We now use Theorem 3.1 to prove a weak approximation theorem.

Theorem 3.4. Let $K$ be a semilocal commutative Hausdorff topological ring, let $F$ be a subring of $K$, let $(A, \sigma, u, \Lambda)$ be a unitary $F$-algebra and let $(P,[f]) \in$ $\mathrm{UQ}^{u, \Lambda}(A, \sigma)$. Assume that:

(0) $\mathbb{F}_{2}$ is not an epimorphic image of $K$,

(1) $K^{\times}$is open in $K$ and the map $a \mapsto a^{-1}: K^{\times} \rightarrow K^{\times}$is continuous, 
(2) $F$ is dense in $K$ and $F \cap K^{\times}=F^{\times}$,

(3) $A$ is a finitely generated projective $F$-module and $\Lambda$ is an $F$-summand of A.

Topologize $\operatorname{End}_{A_{K}}\left(P_{K}\right)$ as in section 2 and give $O\left(\left[f_{K}\right]\right)$ the subspace topology. In addition, define $\mathcal{I}\left(P_{K}\right)$ as in $\mathrm{3B}$ (with $A_{K}$ in the role of $\left.A\right)$. Then, viewing $O([f])$ as a subgroup of $O\left(\left[f_{K}\right]\right)$ via $\phi \mapsto \phi_{K}$, we have

$$
\overline{O([f])} \supseteq \operatorname{ker} \Delta_{\mathcal{I}\left(P_{K}\right),\left[f_{K}\right]} .
$$

In particular, $\left[O\left(\left[f_{K}\right]\right): \overline{O([f])}\right]$ is a finite power of 2 .

Proof. Observe first that condition (3) implies that $A_{K}$ and $\Lambda_{K}$ are projective $K$ modules. Since $P_{K} \in \mathscr{P}\left(A_{K}\right)$, End $A_{K}\left(P_{K}\right)$ is a projective $K$-module and hence can be topologized as in section [2] Furthermore, $\operatorname{End}_{A_{K}}\left(P_{K}\right)^{\times}$is a topological group by Proposition 2.6, so $O\left(\left[f_{K}\right]\right)$ is a topological group as well. Alternatively, we can topologize $O\left(\left[f_{K}\right]\right)$ directly using Proposition 2.1 and the fact that $\mathbf{O}\left(\left[f_{K}\right]\right)$ is defined as an affine $K$-scheme by condition (3). This gives the same topology.

Also note that $A_{K}$ is indeed semilocal: By [24, Cr. II.4.2.4], $A_{K} \operatorname{Jac}(K) \subseteq$ $\operatorname{Jac}\left(A_{K}\right)$. Since $A_{K} / A_{K} \mathrm{Jac}(K)$ is a finitely generated $K / \mathrm{Jac}(K)$-module, it is an artinian ring. It follows that $A_{K} / \operatorname{Jac}\left(A_{K}\right)$ is an epimorphic image of an artinian ring (namely, $A_{K} / A_{K} \mathrm{Jac}(K)$ ), and hence semisimple artinian.

We now turn to the proof itself: By Proposition 3.3. we may apply transfer (see 1E) and hence assume that $P$ is free. Now, by Theorem 3.1 and condition (0), every isometry in ker $\Delta_{\mathcal{I}\left(P_{K}\right)}$ is a product of reflections, so it is enough to show that every neighborhood of a reflection of $\left[f_{K}\right]$ contains a reflection of $[f]$. Indeed, let $s=s_{y, c}$ be a reflection of $\left[f_{K}\right]$. By Proposition 2.7. every neighborhood of $s$ contains a set of the form $\left\{\psi \in O\left(\left[f_{K}\right]\right): \psi x_{i}-s x_{i} \in U_{i} \forall 1 \leq i \leq n\right\}$, where $x_{1}, \ldots, x_{n} \in P_{K}$ and $U_{1}, \ldots, U_{n}$ are neighborhoods of 0 in $P_{K}$. Write $c=\tilde{f}_{K}(y, y)+\gamma$ for $\gamma \in \Lambda_{K}$. Then

$$
s x_{i}=x_{i}-y \cdot\left(\tilde{f}_{K}(y, y)+\gamma\right)^{-1} \cdot \tilde{h}_{f_{K}}\left(y, x_{i}\right) .
$$

By Proposition 2.4. $\tilde{f}_{K}$ and $\tilde{h}_{f_{K}}$ are continuous, and hence, by Propositions 2.5 and 2.6. for each $i$, the function

$$
(y, \gamma) \mapsto x_{i}-y \cdot\left(\tilde{f}_{K}(y, y)+\gamma\right)^{-1} \cdot \tilde{h}_{f_{K}}\left(y, x_{i}\right)
$$

is continuous wherever defined. Furthermore, its domain is an open subset of $P_{K} \times$ $\Lambda_{K}$ by Proposition 2.6(ii) (since $A_{K}^{\times}$is open in $A_{K}$ ). By Proposition 2.8, $P \times \Lambda$ is dense in $P_{K} \times \Lambda_{K}$. Thus, there is $z \in P$ and $\lambda \in \Lambda$ such that $\tilde{f}(z, z)+\lambda \in A_{K}^{\times}$and

$$
\left[x_{i}-y \cdot\left(\tilde{f}_{K}(y, y)+\gamma\right)^{-1} \cdot \tilde{h}_{f_{K}}\left(y, x_{i}\right)\right]-\left[x_{i}-z \cdot(\tilde{f}(z, z)+\lambda)^{-1} \cdot \tilde{h}_{f}\left(z, x_{i}\right)\right] \in U_{i}
$$

for all $i$. Write $d=\tilde{f}(z, z)+\lambda$. Then $d \in A \cap A_{K}^{\times}=A^{\times}$(Proposition [2.9), and hence $s_{z, d}$ is a reflection of $[f]$. Since $s x_{i}-s_{z, d} x_{i} \in U_{i}$ for all $i$, we are done.

Remark 3.5. In the setting of Theorem 3.4

(i) The condition $F \cap K^{\times}=F^{\times}$always holds when $F$ is a field or, more generally, an artinian ring (cf. Remark 2.10).

(ii) The condition that $\Lambda$ is an $F$-summand of $A$ holds when $2 \in A^{\times}$. Indeed, in this case, it is easy to check that $\Lambda=\Lambda^{\min }(u)$ and $A=\Lambda^{\min }(u) \oplus \Lambda^{\min }(-u)$. 
Remark 3.6. Condition (3) of Theorem 3.4 implies that $\mathbf{O}([f])$ is defined as an affine $F$-scheme (see the end of Section 2). At this level or generality, we do not know whether $\Delta_{\mathcal{I}\left(P_{K}\right),\left[f_{K}\right]}: O\left(\left[f_{K}\right]\right) \rightarrow(\mathbb{Z} / 2 \mathbb{Z})^{\mathcal{I}\left(P_{K}\right)}$ is induced from a morphism of affine group schemes over $K$. However, this is true when $K$ is a field: Let $A_{i}$ be a splitorthogonal factor of the semisimple unitary ring $A_{K} / \operatorname{Jac}\left(A_{K}\right)$. The map $O\left(\left[f_{K}\right]\right) \rightarrow$ $O\left(\left[\left(f_{K}\right)_{i}\right]\right)$ is a restriction of the standard map $\operatorname{End}_{A_{K}}\left(P_{K}\right) \rightarrow \operatorname{End}_{A_{i}}\left(P_{i}\right)$, which is $K$-linear. Thus, $O\left(\left[f_{K}\right]\right) \rightarrow O\left(\left[\left(f_{K}\right)_{i}\right]\right)$ is induced by a morphism of affine group schemes over $K$ (cf. Proposition [2.3). Since $\Delta_{\left[\left(f_{K}\right)_{i}\right]}: O\left(\left[\left(f_{K}\right)_{i}\right]\right) \rightarrow \mathbb{Z} / 2 \mathbb{Z}$ is a morphism of algebraic groups over $K$ (see 1F), it follows that $\Delta_{\left[f_{K}\right], i}: O\left(\left[f_{K}\right]\right) \rightarrow$ $\mathbb{Z} / 2 \mathbb{Z}$ is induced by a morphism of affine groups schemes over $K$. Letting $i$ range over $\mathcal{I}\left(P_{K}\right)$, we see that this also holds for $\Delta_{\mathcal{I}\left(P_{K}\right)}: O\left(\left[f_{K}\right]\right) \rightarrow(\mathbb{Z} / 2 \mathbb{Z})^{\mathcal{I}\left(P_{K}\right)}$, where $\mathbb{Z} / 2 \mathbb{Z}$ is viewed as a constant group scheme over $K$.

This argument also works when $K$ is a product of fields $K_{1} \times \cdots \times K_{r}$. However, in this case, $(\mathbb{Z} / 2 \mathbb{Z})^{\mathcal{I}\left(P_{K}\right)}$ should be realized as $\prod_{j=1}^{r}(\mathbb{Z} / 2 \mathbb{Z})^{\mathcal{I}\left(P_{K_{j}}\right)}$ where $(\mathbb{Z} / 2 \mathbb{Z})^{\mathcal{I}\left(P_{K_{j}}\right)}$ is a constant group scheme over $K_{j}$.

The following corollary shows that weak approximation holds for the connected component of $\mathbf{O}([f])$ when $F$ is a field (see [38, $\S 6.7]$ for the definition).

Corollary 3.7. Keep the setting of Theorem 3.4, and assume further that $F$ is a field and $K$ is a product of finitely many fields. Let $\mathbf{O}^{+}:=\mathbf{O}([f])^{+}$be the connected component of $\mathbf{O}:=\mathbf{O}([f])$. Give $\mathbf{O}^{+}(K)$ the topology of Proposition 2.1 . Then $\mathbf{O}^{+}(F)$ is dense in $\mathbf{O}^{+}(K)$.

Proof. We will use of the algebraic group $\pi_{0}(\mathbf{O})$; see [38, §6.5] for its definition. The exact sequence of algebraic groups $1 \rightarrow \mathbf{O}^{+} \rightarrow \mathbf{O} \rightarrow \pi_{0}(\mathbf{O}) \rightarrow 1$ gives rise to an exact sequence $1 \rightarrow \mathbf{O}^{+}(K) \rightarrow \mathbf{O}(K) \rightarrow \pi_{0}(\mathbf{O})(K)$ and all the morphisms are continuous by Proposition 2.1. Since $\pi_{0}(\mathbf{O})(K)$ is finite and $K$ is Hausdorff, $\pi_{0}(\mathbf{O})(K)$ is discrete, and hence $\mathbf{O}^{+}(K)$ is open and closed in $\mathbf{O}(K)$.

By Remark 3.6, the map $\Delta_{\mathcal{I}\left(P_{K}\right)}$ is induced from a homomorphism of algebraic groups $\Delta: \mathbf{O} \rightarrow(\mathbb{Z} / 2 \mathbb{Z})^{\mathcal{I}\left(P_{K}\right)}$. Furthermore, $(\mathbb{Z} / 2 \mathbb{Z})^{\mathcal{I}\left(P_{K}\right)}$ is étale over Spec $K$, and hence $\triangleq$ factors through $\mathbf{O} \rightarrow \pi_{0}(\mathbf{O})$ [38, Th. 6.7]. It follows that $\mathbf{O}^{+}(K) \subseteq$ ker $\Delta_{\mathcal{I}\left(P_{K}\right)}$. By Theorem 3.4, this implies $\mathbf{O}^{+}(K) \subseteq \overline{\mathbf{O}(F)}$, and since $\mathbf{O}^{+}(K)$ is open and closed in $\mathbf{O}(K)$, we get $\mathbf{O}^{+}(K)=\overline{\mathbf{O}(F) \cap \mathbf{O}^{+}(K)}=\overline{\mathbf{O}^{+}(F)}$.

\section{A double coset ARgument}

Given a commutative ring $R$, a family of commutative $R$-algebras $\mathcal{L}$, and a quadratic space $(P,[f])$ over a unitary $R$-algebra, we let the $\mathcal{L}$-genus of $(P,[f])$, denoted $\operatorname{gen}_{\mathcal{L}}(P,[f])$, be the collection of isomorphism classes of quadratic spaces $\left(P^{\prime},\left[f^{\prime}\right]\right)$ that become isometric to $(P,[f])$ after applying $\mathcal{R}_{L / R}$ for every $L \in \mathcal{L}$. This generalizes the genus considered in the introduction.

As preparation for the patching theorem of the next section, this section relates the $\mathcal{L}$-genus of $(P,[f])$ to double cosets in a certain group, in case $\mathcal{L}$ consists of two algebras satisfying certain assumptions. The argument resembles some descent methods used in number theory and algebraic geometry, which generalize much beyond the scope of quadratic spaces. We comment about this in detail in Remark 4.6. 
Recall that a commutative square of abelian groups

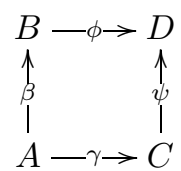

is cartesian if $A$ is the pullback of $\phi$ and $\psi$. Namely, for all $b \in B, c \in C$ with $\phi b=\psi c$, there exists unique $a \in A$ with $\beta a=b$ and $\gamma a=c$. We say that the square is onto if $\phi(B)+\psi(C)=D$.

The properties just defined can be explained via exactness of the following sequence

$$
0 \rightarrow A \stackrel{a \mapsto(\beta a \oplus \gamma a)}{\longrightarrow} B \oplus C \stackrel{(b \oplus c) \mapsto(\phi b-\psi c)}{\longrightarrow} D \rightarrow 0 .
$$

Namely, the square (4.1) is cartesian if the sequence is exact on the left and in the middle, and onto if it is exact on the right.

Throughout, we fix a cartesian and onto square of commutative rings:

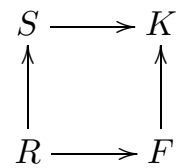

In addition, $(A, \sigma, u, \Lambda)$ is a unitary $R$-algebra such that $A$ is flat as an $R$-module.

We shall use the notation of $1 \mathrm{C}$ for scalar extension of modules, homomorphisms, quadratic forms, etc. Furthermore, for brevity, for all $P \in \mathscr{P}(A)$, we set

$$
\mathcal{S}(P):=\operatorname{Hom}\left(P, P^{*}\right) \quad \text { and } \quad \mathcal{Q}(P)=\operatorname{Hom}\left(P, P^{*}\right) / \Lambda_{P} .
$$

Similar notation will be used for modules over $A_{S}, A_{F}$ and $A_{K}$. Recall from $1 \mathrm{C}$ that we have scalar extension maps

$$
f \mapsto f_{S}: \mathcal{S}(P) \rightarrow \mathcal{S}\left(P_{S}\right), \quad[f] \mapsto\left[f_{S}\right]: \mathcal{Q}(P) \rightarrow \mathcal{Q}\left(P_{S}\right)
$$

and likewise for any pair of the rings $R, S, F, K$ connected by a homomorphism.

Lemma 4.1. For any flat $R$-module $M$, the following square is cartesian and onto:

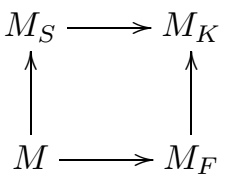

Proof. As explained above, the square (4.2) gives rise to an exact sequence

$$
0 \rightarrow R \rightarrow S \oplus K \rightarrow F \rightarrow 0 .
$$

The lemma follows by tensoring with $M$, which preserves exactness since $M$ is flat.

Lemma 4.2. Let $P, P^{\prime} \in \mathscr{P}(A)$. Consider the following squares induced by (4.2):
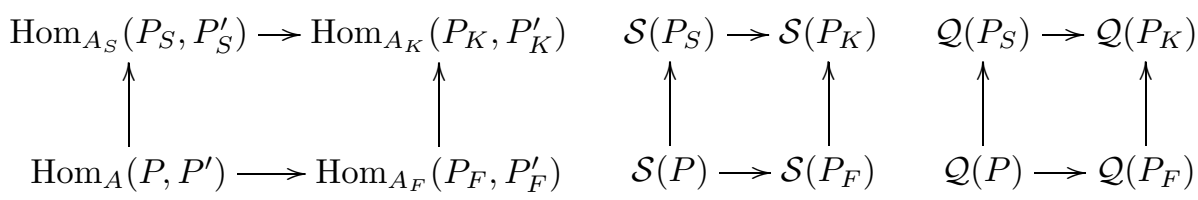
Then:

(i) The left and middle squares are cartesian and onto. Furthermore, if $\psi \in$ $\operatorname{Hom}_{A}\left(P, P^{\prime}\right)$ is such that $\psi_{F}$ and $\psi_{S}$ are invertible, then $\psi$ is invertible.

(ii) Provided $K$ is flat as an $R$-module, the right square is cartesian and onto.

Proof. (i) By Lemma 1.2 the middle square is a special case of the left square. This lemma also allows us to identify $\operatorname{Hom}_{A_{T}}\left(P_{T}, P_{T}^{\prime}\right)$ with $\operatorname{Hom}_{A}\left(P, P^{\prime}\right)_{T}$ for any commutative $R$-algebra $T$. Now, by Lemma 4.1, in order to prove that the left square is cartesian and onto, it is enough to show that $\operatorname{Hom}_{A}\left(P, P^{\prime}\right)$ is a flat $R$ module. Indeed, $\operatorname{Hom}_{A}\left(P, P^{\prime}\right)$ is a summand of $\operatorname{Hom}_{A}\left(A^{n}, A^{m}\right) \cong A^{n m}$ (as $R$ modules) for some $n, m \in \mathbb{N}$, and $A$ is flat by assumption.

Next, assume $\psi \in \operatorname{Hom}_{A}\left(P, P^{\prime}\right)$ is such that $\psi_{F}$ and $\psi_{S}$ are invertible. Then in $\operatorname{Hom}_{A_{K}}\left(P_{K}^{\prime}, P_{K}\right)$ we have $\left(\psi_{S}^{-1}\right)_{K}=\left(\psi_{F}^{-1}\right)_{K}$, hence there exists $\phi \in \operatorname{Hom}_{A}\left(P^{\prime}, P\right)$ with $\phi_{S}=\psi_{S}^{-1}$ and $\phi_{F}=\psi_{F}^{-1}$. We clearly have $\left(\phi \psi-\operatorname{id}_{P}\right)_{S}=0$ and $\left(\phi \psi-\operatorname{id}_{P}\right)_{F}=$ 0 , so by cartesianity of the left square (in case $P=P^{\prime}$ ), we have $\phi \psi=\mathrm{id}_{P}$. Likewise, $\psi \phi=\operatorname{id}_{P^{\prime}}$, so $\psi$ is invertible.

(ii) By Proposition [1.3, we may identify $\mathcal{S}\left(P_{T}\right)$ with $\mathcal{S}(P)_{T}$ and $\mathcal{Q}\left(P_{T}\right)$ with $\mathcal{Q}(P)_{T}$ for any $R$-algebra $T$. Consider the following diagram, which is obtained by tensoring the top row with (4.4):

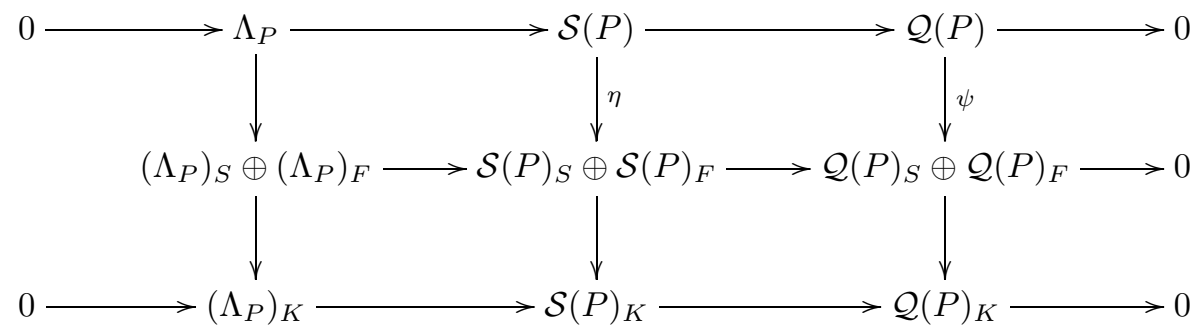

The first two rows are clearly exact, and the third row is exact since $K$ is flat. In addition, all columns are exact in the middle and on the bottom (once adding a zero object), and by (i), $\eta$ is injective. We only need to prove that $\psi$ is injective, and this follows by standard diagram chasing. (Specifically, assume $a \in \mathcal{Q}(P)$ satisfies $\psi a=0$. Let $b \in \mathcal{S}(P)$ be an inverse image of $a$ and let $c=\eta b$. Then the image of $c$ in $\mathcal{Q}(P)_{S} \oplus \mathcal{Q}(P)_{F}$ is $\psi a=0$, hence $c$ has an inverse image $d \in\left(\Lambda_{P}\right)_{S} \oplus\left(\Lambda_{P}\right)_{F}$. The image of $d$ in $\mathcal{S}(P)_{K}$ is the image of $c=\eta b$ in $\mathcal{S}(P)_{K}$, which is 0 . Thus, the image of $d$ in $\left(\Lambda_{P}\right)_{K}$ is 0 (since the third row is exact on the left). Let $e \in \Lambda_{P}$ be an inverse image of $d$, and let $f$ be the image of $e$ in $\mathcal{S}(P)$. Then $\eta f=c=\eta b$, so $f=b$ (since $\eta$ is injective). This means that $a$ is the image of $e \in \Lambda_{P}$, and hence equals 0.$)$

We remark that part (i) of the lemma implies the following corollary.

Corollary 4.3. Let $(P,[f])$ be a quadratic space over $(A, \sigma, u, \Lambda)$ and let $\left(P^{\prime},\left[f^{\prime}\right]\right) \in$ $\operatorname{gen}_{S, F}(P,[f])$. If $(P,[f])$ is unimodular, then so is $\left(P^{\prime},\left[f^{\prime}\right]\right)$.

Proof. Write $h=h_{f}$ and $h^{\prime}=h_{f^{\prime}}$. Then both $h_{S}$ and $h_{F}$ are invertible, hence $h_{S}^{\prime}$ and $h_{F}^{\prime}$ are invertible (because $\left.\left(P^{\prime},\left[f^{\prime}\right]\right) \in \operatorname{gen}_{S, F}(P,[f])\right)$. Thus, by Lemma 4.2(i), $h^{\prime}$ is invertible. 
Notation 4.4. Let $(P,[f])$ be a quadratic space over $(A, \sigma, u, \Lambda)$. We set:

$$
\begin{aligned}
O_{S} & =\left\{\phi_{K} \mid \phi \in O\left(\left[f_{S}\right]\right)\right\}, & & G_{S}=\left\{\phi_{K} \mid \phi \in \operatorname{End}_{A_{S}}\left(P_{S}\right)^{\times}\right\}, \\
O_{F} & =\left\{\phi_{K} \mid \phi \in O\left(\left[f_{F}\right]\right)\right\}, & & G_{F}=\left\{\phi_{K} \mid \phi \in \operatorname{End}_{A_{F}}\left(P_{F}\right)^{\times}\right\}, \\
O_{K} & =O\left(P_{K},\left[f_{K}\right]\right), & & G_{K}=\operatorname{End}_{A_{K}}\left(P_{K}\right)^{\times} .
\end{aligned}
$$

Theorem 4.5. Let $(P,[f])$ be a quadratic space over $(A, \sigma, u, \Lambda)$, and assume that $K$ is a flat $R$-module. Then, in the notation of 4.4 , there exists an injection

$$
\Phi: \operatorname{gen}_{S, F}(P,[f]) \rightarrow O_{S} \backslash O_{K} / O_{F},
$$

constructed in the proof. When $O_{K} \subseteq G_{S} G_{F}$, the map $\Phi$ is bijective and every $\left(P^{\prime},\left[f^{\prime}\right]\right) \in \operatorname{gen}_{S, F}(P,[f])$ satisfies $P^{\prime} \cong P$.

Proof. We shall use the following special notation: If $(Q,[g])$ is a quadratic space and $\phi \in \operatorname{Hom}\left(Q^{\prime}, Q\right)$, define

$$
[g] \bullet \phi=\left[\phi^{*} g \phi\right] .
$$

We clearly have $[g] \bullet(\phi \psi)=([g] \bullet \phi) \bullet \psi$ when both sides are well-defined.

We first construct $\Phi$. Let $\left(P^{\prime},\left[f^{\prime}\right]\right)$ be a representative for an isomorphism class in $\operatorname{gen}_{S, F}(P,[f])$. Then there are isometries $\phi:\left(P_{S}^{\prime},\left[f_{S}^{\prime}\right]\right) \rightarrow\left(P_{S},\left[f_{S}\right]\right)$ and $\psi:$ $\left(P_{F}^{\prime},\left[f_{F}^{\prime}\right]\right) \rightarrow\left(P_{F},\left[f_{F}\right]\right)$, and we have $\phi_{K} \psi_{K}^{-1} \in O_{K}$. Using this, define

$$
\Phi\left(P^{\prime},\left[f^{\prime}\right]\right)=O_{S} \phi_{K} \psi_{K}^{-1} O_{F} .
$$

Observe that $\Phi\left(P^{\prime},\left[f^{\prime}\right]\right)$ is independent of the choices of $\phi$ and $\psi$. Indeed, if $\theta$ : $\left(P_{S}^{\prime},\left[f_{S}^{\prime}\right]\right) \rightarrow\left(P_{S},\left[f_{S}\right]\right)$ and $\xi:\left(P_{F}^{\prime},\left[f_{F}^{\prime}\right]\right) \rightarrow\left(P_{F},\left[f_{F}\right]\right)$ are other isometries, then $\phi_{K} \theta_{K}^{-1} \in O_{S}$ and $\psi_{K} \xi_{K}^{-1} \in O_{F}$, hence we get

$$
O_{S} \theta_{K} \xi_{K}^{-1} O_{F}=O_{S}\left(\phi_{K} \theta_{K}^{-1}\right) \theta_{K} \xi_{K}^{-1}\left(\psi_{K} \xi_{K}^{-1}\right)^{-1} O_{F}=O_{S} \phi_{K} \psi_{K}^{-1} O_{F}
$$

To see that $\Phi$ is well-defined up to isometry (over $R$ ), let $\eta:\left(P^{\prime \prime},\left[f^{\prime \prime}\right]\right) \rightarrow\left(P^{\prime},\left[f^{\prime}\right]\right)$ be an isometry. Then there are isometries $\phi \eta_{S}:\left(P_{S}^{\prime \prime},\left[f_{S}^{\prime \prime}\right]\right) \rightarrow\left(P_{S},\left[f_{S}\right]\right)$ and $\psi \eta_{F}$ : $\left(P_{F}^{\prime \prime},\left[f_{F}^{\prime \prime}\right]\right) \rightarrow\left(P_{F},\left[f_{F}\right]\right)$. We now have

$$
\Phi\left(P^{\prime \prime},\left[f^{\prime \prime}\right]\right)=O_{S}\left(\phi \eta_{S}\right)_{K}\left(\psi \eta_{F}\right)_{K}^{-1} O_{F}=O_{S} \phi_{K} \psi_{K}^{-1} O_{F}=\Phi\left(P^{\prime},\left[f^{\prime}\right]\right),
$$

as required.

Next, we verify that $\Phi$ is injective. Assume that $\Phi\left(P^{\prime},\left[f^{\prime}\right]\right)=\Phi\left(P^{\prime \prime},\left[f^{\prime \prime}\right]\right)$, let $\phi, \psi$ be as above, and let $\phi^{\prime}:\left(P_{S}^{\prime \prime},\left[f_{S}^{\prime \prime}\right]\right) \rightarrow\left(P_{S},\left[f_{S}\right]\right), \psi^{\prime}:\left(P_{F}^{\prime \prime},\left[f_{F}^{\prime \prime}\right]\right) \rightarrow\left(P_{F},\left[f_{F}\right]\right)$. Then

$$
O_{S} \phi_{K} \psi_{K}^{-1} O_{K}=O_{S} \phi_{K}^{\prime} \psi_{K}^{\prime-1} O_{F},
$$

so we can write $\phi_{K} \psi_{K}^{-1}=\alpha_{K} \phi_{K}^{\prime} \psi_{K}^{\prime-1} \beta_{K}^{-1}$ for $\alpha \in O\left(P_{S},\left[f_{S}\right]\right)$ and $\beta \in O\left(P_{F},\left[f_{F}\right]\right)$. This implies

$$
\left(\phi^{-1} \alpha \phi^{\prime}\right)_{K}=\phi_{K}^{-1} \alpha_{K} \phi_{K}^{\prime}=\psi_{K}^{-1} \beta_{K} \psi_{K}^{\prime}=\left(\psi^{-1} \beta \psi^{\prime}\right)_{K} .
$$

By Lemma 4.2(i), there exists an isomorphism $\eta: P^{\prime \prime} \rightarrow P^{\prime}$ with

$$
\eta_{S}=\phi^{-1} \alpha \phi^{\prime} \quad \text { and } \quad \eta_{F}=\psi^{-1} \beta \psi^{\prime} \text {. }
$$

We now have

$$
\left(\left[f^{\prime}\right] \bullet \eta\right)_{S}=\left[f_{S}^{\prime}\right] \bullet\left(\phi^{-1} \alpha \phi^{\prime}\right)=\left[f_{S}\right] \bullet\left(\alpha \phi^{\prime}\right)=\left[f_{S}\right] \bullet \phi^{\prime}=\left[f_{S}^{\prime \prime}\right]
$$

and likewise, $\left(\left[f^{\prime}\right] \bullet \eta\right)_{F}=\left[f_{F}^{\prime \prime}\right]$. By Lemma 4.2(ii), this means $\left[f^{\prime}\right] \bullet \eta=\left[f^{\prime \prime}\right]$, so $\left(P^{\prime},\left[f^{\prime}\right]\right) \cong\left(P^{\prime \prime},\left[f^{\prime \prime}\right]\right)$.

Assume now that $O_{K} \subseteq G_{S} G_{F}$. We will show that $\Phi$ is onto and, moreover, that every double coset $O_{S} \eta O_{F}$ arises from a quadratic form defined on $P$. Let 
$O_{S} \eta O_{F}$ be a double coset in $O_{K}$. Since $O_{K} \subseteq G_{S} G_{F}$, we can write $\eta=\alpha_{K} \beta_{K}^{-1}$ with $\alpha \in \operatorname{End}_{A_{S}}\left(P_{S}\right)^{\times}$and $\beta \in \operatorname{End}_{A_{F}}\left(P_{F}\right)^{\times}$. Since $\alpha_{K} \beta_{K}^{-1} \in O_{K}$, we have

$$
\left(\left[f_{S}\right] \bullet \alpha\right)_{K}=\left[f_{K}\right] \bullet \alpha_{K}=\left[f_{K}\right] \bullet\left(\alpha_{K} \beta_{K}^{-1} \beta_{K}\right)=\left[f_{K}\right] \bullet \beta_{K}=\left(\left[f_{F}\right] \bullet \beta\right)_{K} .
$$

By Lemma 4.2 (ii), there exists unique $[g] \in \mathcal{Q}(P)$ with

$$
\left[g_{S}\right]=\left[f_{S}\right] \bullet \alpha \quad \text { and } \quad\left[g_{F}\right]=\left[f_{F}\right] \bullet \beta .
$$

We clearly have $(P,[g]) \in \operatorname{gen}_{S, F}(P,[f])$, and $\Phi(P,[g])=O_{S} \alpha_{K} \beta_{K}^{-1} O_{F}=O_{S} \eta O_{F}$ by the definition of $\Phi$, as required.

Remark 4.6. At this level of generality, we do not know if the map $\Phi$ is onto. In [24, I. 11], Knus calls the square (4.2) a patching diagram for quadratic modules when $\Phi$ is an isomorphism, and several other conditions are satisfied.

One can use descent theory to show that $\Phi$ is an isomorphism in certain cases, even without assuming $O_{K} \subseteq G_{S} G_{F}$. This typically requires $S \oplus F$ to be faithfully flat, $K=S \otimes_{R} F$, and one must show that any $\psi \in O_{K}$, which we view as $\psi:\left[\left(f_{S}\right)_{F}\right] \rightarrow\left[\left(f_{F}\right)_{S}\right]$ can be completed to a descent data (i.e. a family of isometries $\psi_{T, Z}:\left[\left(f_{T}\right)_{Z}\right] \rightarrow\left[\left(f_{Z}\right)_{T}\right]$ for $T, Z \in\{F, S\}$ satisfying the cocycle condition; see [11, p. 132], for instance). For certain families of squares as in (4.2), this problem was studied extensively in the literature under the broader context of torsors of group schemes. See [13, Pr. 2.6] for a general result of this kind. These methods seem to go back at least as far as [16, Apx.].

We further comment that the double cosets considered in Theorem 4.5 are strongly related with Nisnevich cohomology of affine group schemes over Dedekind domains. Specifically, let $R$ be a Dedekind domain, $F$ its fraction field, $S$ the product of its (non-archimedean) completions, and $K$ the ring of (finite) adeles of $R$. Then for any affine group scheme $\mathbf{G}$ over $R$ satisfying certain mild assumptions, the double cosets $\mathbf{G}(S) \backslash \mathbf{G}(K) / \mathbf{G}(F)$ are in correspondence with the first Nisnevich cohomology $\mathrm{H}_{\mathrm{Nis}}^{1}(R, \mathbf{G})$; see [27, Th. 3.5] and also [28, Th. 2.1], [18, Apx.].

Remark 4.7. Theorem 4.5 and also Theorem 5.1 below, do not assume that $\mathbf{O}([f])$ is defined as an affine $R$-scheme. For example, consider the cases

- $(A, \sigma, u, \Lambda)=\left(\left[\begin{array}{ll}\mathbb{Z} & \mathbb{Q} \\ \mathbb{Z}\end{array}\right],\left[\begin{array}{cc}a & b \\ d\end{array}\right] \mapsto\left[\begin{array}{ll}d & b \\ a\end{array}\right], 1, \Lambda^{\min }(1)\right)$ or

- $(A, \sigma, u, \Lambda)=(\mathbb{Z}$, id $,-1,2 \mathbb{Z})$,

with $R=\mathbb{Z}$. Theorem 4.5 would apply (with suitable $S, F, K$ ), but it is not clear to us whether $\mathbf{O}([f])$ is defined as an affine $R$-scheme.

\section{Patching}

We now state and prove a patching theorem for quadratic spaces.

Theorem 5.1. Let $K$ be a commutative semilocal topological ring, let $S, F \subseteq K$ be subrings of $K$, let $R=S \cap F$, let $(A, \sigma, u, \Lambda)$ be a unitary $R$-algebra, and let $(P,[f]) \in \mathrm{UQ}^{u, \Lambda}(A, \sigma)$. Assume that:

(0) $\mathbb{F}_{2}$ is not an epimorphic image of $K$,

(1) $K^{\times}$is open in $K$ and the map $a \mapsto a^{-1}: K^{\times} \rightarrow K^{\times}$is continuous,

(2) $F$ is dense in $K$ and $F \cap K^{\times}=F^{\times}$,

(3) $A_{F} \in \mathscr{P}(F)$ and $\Lambda_{F}$ is a summand of $A_{F}$,

(4) $K$ and $A$ are flat $R$-modules,

(5) $S$ is open in $K$. 
Let $\mathcal{I}=\mathcal{I}\left(P_{K}\right)$ and $\Delta:=\Delta_{\mathcal{I},\left[f_{K}\right]}($ see $3 \mathrm{~A}$, 3B] $)$. Then

$$
\left|\operatorname{gen}_{S, F}(P,[f])\right|=\frac{\left|\Delta\left(O\left(\left[f_{K}\right]\right)\right)\right|}{\left|\Delta\left(O\left(\left[f_{F}\right]\right)\right)+\Delta\left(O\left(\left[f_{S}\right]\right)\right)\right|} .
$$

In addition, for all $\left(P^{\prime},\left[f^{\prime}\right]\right) \in \operatorname{gen}_{S, F}(P,[f])$, we have $P \cong P^{\prime}$.

Before giving the proof, let us present an example in which the theorem can be applied, and the $\{S, F\}$-genus can be given a more concrete meaning.

Example 5.2. Suppose $R$ is a Dedekind domain with finitely many ideals (or equivalently, $R$ is a semilocal PID). For $\mathfrak{p} \in \operatorname{Spec} R$, denote by $\hat{R}_{\mathfrak{p}}$ the completion of $R_{\mathfrak{p}}$ (which is a discrete valuation ring) and let $\hat{F}_{\mathfrak{p}}$ denote the fraction field of $\hat{R}_{\mathfrak{p}}$. Note that $F:=\hat{R}_{0}$ is just the fraction field of $R$. We endow $\hat{R}_{\mathfrak{p}}$ and $\hat{F}_{\mathfrak{p}}$ with their natural topologies. Now, let

$$
S=\prod_{0 \neq \mathfrak{p}} \hat{R}_{\mathfrak{p}} \quad \text { and } \quad K=\prod_{0 \neq \mathfrak{p}} \hat{F}_{\mathfrak{p}} .
$$

We embed $F$ diagonally in $K$. It it well-known that $F$ is dense in $K$ [15, Th. 11.6], and $F \cap S=R$. Furthermore, any torsion-free $R$-module is flat [26, Th. 4.69]. This means that Theorem 5.1 can be applied with any unitary $R$-algebra $(A, \sigma, u, \Lambda)$ such that $A$ is torsion-free and $\operatorname{dim}_{F} A_{F}<\infty$. Moreover, for $(P,[f]) \in \mathrm{UQ}^{u, \Lambda}(A, \sigma)$, we have

$$
\operatorname{gen}_{S, F}(P,[f])=\operatorname{gen}_{\left\{\hat{R}_{\mathfrak{p}} \mid \mathfrak{p} \in \operatorname{Spec} R\right\}}(P,[f]) .
$$

That is, the $\{S, F\}$-genus is the genus considered in the introduction.

Now, let $k(\mathfrak{p})$ denote the residue field at $\mathfrak{p}$, namely, $R_{\mathfrak{p}} / \mathfrak{p}_{\mathfrak{p}}$. If $A$ is finitely generated and projective as an $R$-module, then a standard lifting argument (see [30, Th. 2.2(2)] or [24, Th. II.4.6.1], for instance), implies that $\mathcal{R}_{k(\mathfrak{p}) / R}(P,[f]) \cong$ $\mathcal{R}_{k(\mathfrak{p}) / R}\left(P^{\prime},\left[f^{\prime}\right]\right)$ if and only if $\mathcal{R}_{\hat{R}_{\mathfrak{p}} / R}(P,[f]) \cong \mathcal{R}_{\hat{R}_{\mathfrak{p}} / R}\left(P^{\prime},\left[f^{\prime}\right]\right)$. Thus, in this case, $\operatorname{gen}_{S, F}(P,[f])=\operatorname{gen}_{\{k(\mathfrak{p}) \mid \mathfrak{p} \in \operatorname{Spec} R\}}(P,[f])$.

If in addition $\Lambda$ is a summand of $A$, then $\mathbf{O}([f])$ is defined as an affine $R$-scheme (see the appendix) and $\operatorname{gen}_{S, F}(P,[f])$ corresponds to the first Nisnevich cohomology $\mathrm{H}_{\mathrm{Nis}}^{1}(R, \mathbf{O}([f]))$ (cf. Remark 4.6]).

The proof of Theorem 5.1 requires a technical lemma. As in section 3, we topologize all objects defined over $K$.

Lemma 5.3. Keep the assumptions of Theorem 5.1 and let $P^{\prime} \in \mathscr{P}(A)$. Then the maps (cf. 1C)

$$
\begin{aligned}
& \mathcal{R}_{K / S}: \operatorname{Hom}_{A_{S}}\left(P_{S}, P_{S}^{\prime}\right) \rightarrow \operatorname{Hom}_{A_{K}}\left(P_{K}, P_{K}^{\prime}\right), \\
& \mathcal{R}_{K / S}: \operatorname{End}_{A_{S}}\left(P_{S}\right)^{\times} \rightarrow \operatorname{End}_{A_{K}}\left(P_{K}\right)^{\times}, \\
& \mathcal{R}_{K / S}: \Lambda_{P_{S}} \rightarrow \Lambda_{P_{K}}, \\
& \mathcal{R}_{K / S}: O\left(\left[f_{S}\right]\right) \rightarrow O\left(\left[f_{K}\right]\right),
\end{aligned}
$$

are injective, and their image is open (in the appropriate topology).

Proof. When $A \in \mathscr{P}(R)$ and $\Lambda$ is a summand of $A, \mathbf{O}([f])$ is defined as an affine $R$-scheme (see the appendix), and then the lemma follows from 14, Ex. 2.2].

We prove the general case directly: By Lemma 1.2, we may identify End $_{A_{T}}\left(P_{T}, P_{T}^{\prime}\right)$ with $\operatorname{End}_{A}\left(P, P^{\prime}\right)_{T}$ and $\operatorname{Hom}_{A_{T}}\left(P_{T},\left(P_{T}\right)^{*}\right)$ with $\operatorname{Hom}_{A}\left(P, P^{*}\right)_{T}$ 
for any $R$-algebra $T$. As in the proof of Lemma 4.2(i), $\operatorname{End}_{A}\left(P, P^{\prime}\right)$ is a flat $R$ module. Thus, $\mathcal{R}_{K / S}: \operatorname{Hom}_{A}\left(P, P^{\prime}\right)_{S} \rightarrow \operatorname{Hom}_{A}\left(P, P^{\prime}\right)_{K}$ is an injection (because the inclusion map $S \rightarrow K$ is an injection). The other three maps in the lemma are just restrictions of $\mathcal{R}_{K / S}: \operatorname{Hom}_{A_{S}}\left(P_{S}, P_{S}^{\prime}\right) \rightarrow \operatorname{Hom}_{P_{K}}\left(P_{K}, P_{K}^{\prime}\right)$ in the special cases $P^{\prime}=P^{*}$ and $P^{\prime}=P$, so they are also injective.

We identify $\operatorname{Hom}_{A_{S}}\left(P_{S}, P_{S}^{\prime}\right)$ and $\Lambda_{P_{S}}$ with their images in $\operatorname{Hom}_{P_{K}}\left(P_{K}, P_{K}^{\prime}\right)$ and $\Lambda_{P_{K}}$, respectively. To prove that these images are open, it is enough to prove the following general claim: Let $Q$ be an f.g. projective $K$-module. Then any $S$ submodule $Q^{\prime} \subseteq Q$ with $Q^{\prime} \cdot K=Q$ is open $Q$. (Note that we have $\operatorname{Hom}_{A}\left(P, P^{\prime}\right)_{S}$. $K=\operatorname{Hom}_{A}\left(P, P^{\prime}\right)_{K}$ by definition, and $\Lambda_{P_{S}} \cdot K=\Lambda_{P_{K}}$ by Proposition 1.3.) Indeed, $Q^{\prime}$ contains a finite set $\left\{x_{i}\right\}_{i=1}^{t}$ generating $Q$. It is well known that there are $\left\{\psi_{i}\right\}_{i=1}^{t} \subseteq Q^{\vee}=\operatorname{Hom}_{K}(Q, K)$ such that $\left\{x_{i}, \psi_{i}\right\}_{i=1}^{t}$ is a dual basis for $Q$. (See the proof of Proposition 2.7 for the definition; construct the $\psi_{i}$ 's by a section of the projection $\bigoplus_{i} K \rightarrow Q$ given by $\bigoplus_{i} k_{i} \mapsto \sum_{i} x_{i} k_{i}$.) Each $\psi_{i}: Q \rightarrow K$ is $K$ linear, hence continuous, and thus $\bigcap_{i=1}^{t} \psi_{i}^{-1}(S)$ is open (because $S$ is open in $K$ ). However, for all $x \in \bigcap_{i} \psi_{i}^{-1}(S)$, we have $x=\sum_{i} x_{i}\left(\psi_{i} x\right) \in \sum_{i} x_{i} S \subseteq Q^{\prime}$. Thus, $Q^{\prime}$ contains an open $S$-submodule, so $Q^{\prime}$ is open in $Q$.

Next, we show that $\operatorname{End}_{A_{S}}\left(P_{S}\right)^{\times}$is open in $\operatorname{End}_{A_{K}}\left(P_{K}\right)^{\times}$: Write $E_{S}=\operatorname{End}_{A_{S}}\left(P_{S}\right)$ and $E_{K}=\operatorname{End}_{A_{K}}\left(P_{K}\right)$. By what we have shown above, $E_{S}$ is open in $E_{K}$. Thus, $E_{S} \cap E_{K}^{\times}$is open in $E_{K}^{\times}$. By Proposition 2.6 (ii), $\left(E_{S} \cap E_{K}^{\times}\right)^{-1}$ is also open in $E_{K}^{\times}$, hence $E_{S} \cap\left(E_{S} \cap E_{K}^{\times}\right)^{-1}$ is open as well. But this set is $E_{S}^{\times}$.

Finally, $O\left(\left[f_{S}\right]\right)$ is open in $O\left(\left[f_{K}\right]\right)$ because $O\left(\left[f_{S}\right]\right)=O\left(\left[f_{K}\right]\right) \cap E_{S}^{\times}$.

Proof of Theorem 5.1. We shall make use of the following well-known fact about topological groups:

(*) If $G$ is a topological (multiplicative) group and $X$ is any subset of $G$, then $\bar{X}=\bigcap_{U} X U$, where $U$ ranges over all neighborhoods of $1_{G}$.

A proof can be found in [37, Th. 3.3(3)] (the proof is given for abelian groups but generalizes verbatim to non-abelian groups). Note that $(*)$ implies that for any neighborhood $U$ of $1_{G}$, we have $\bar{X} \subseteq X U$. We henceforth use the notation of 4.4 .

It is clear that the rings $R, S, F, \bar{K}$ form a cartesian square as in section 4 . This square is onto since $S+F \supseteq \bar{F}=K$ by $(*)$. That $A$ and $K$ are flat over $R$ holds by assumption. Furthermore, we claim that $O_{K} \subseteq G_{S} G_{F}$. Indeed, by Lemma 5.3, $G_{S}$ is open in $G_{K}$, and by Proposition 2.9. $G_{F}$ is dense in $G_{K}$. Thus, by (*), $G_{S} G_{F} \supseteq \overline{G_{F}}=G_{K}$, as required. We may therefore apply Theorem 4.5 to get a one-to-one correspondence

$$
\operatorname{gen}_{S, F}(P,[f]) \longleftrightarrow O_{S} \backslash O_{K} / O_{F}
$$

We also get that $\left(P^{\prime},\left[f^{\prime}\right]\right) \in \operatorname{gen}_{S, F}(P,[f])$ implies $P^{\prime} \cong P$.

Let $H_{K}=\Delta\left(O_{K}\right), H_{S}=\Delta\left(O_{S}\right)$ and $H_{F}=\Delta\left(O_{F}\right)$. We will prove the theorem by constructing a bijection between $O_{S} \backslash O_{K} / O_{F}$ and $H_{K} /\left(H_{S}+H_{F}\right)$, whose size is clearly the desired quantity. Define $\Psi: O_{S} \backslash O_{K} / O_{F} \rightarrow H_{K} /\left(H_{S}+H_{F}\right)$ by

$$
\Psi\left(O_{S} \phi O_{F}\right)=\Delta(\phi)+H_{S}+H_{F} .
$$

It is clear that $\Psi$ is well-defined and surjective. To show $\Psi$ is injective, it is enough to prove that $\Delta^{-1}\left(\Delta(\phi)+H_{S}+H_{F}\right)=O_{S} \phi O_{F}$.

Let $\phi \in O_{K}$ and let $t=\Delta(\phi)$. We claim that $\Delta^{-1}(t) \subseteq O_{S} \phi O_{F}$. Indeed, by Theorem 3.4 (whose assumptions hold by conditions (0)-(3)), ker $\Delta \subseteq \overline{O_{F}}$. Thus, $\Delta^{-1}(t)=\phi \cdot \operatorname{ker} \Delta \subseteq \phi \overline{O_{F}}=\overline{\phi O_{F}}$ and $\overline{\phi O_{F}} \subseteq O_{S} \phi O_{F}$ by $(*)$. 
Now let $x \in H_{S}$ and $y \in H_{F}$. Then there are $\psi \in O_{S}, \eta \in O_{F}$ with $\Delta(\psi)=x$ and $\Delta(\eta)=y$. By the previous paragraph, we have $\Delta^{-1}(x+t+y) \subseteq O_{S} \psi \phi \eta O_{F}=$ $O_{S} \phi O_{F}$, so $\Delta^{-1}\left(\Delta(\phi)+H_{S}+H_{F}\right)=\bigcup_{x \in H_{S}, y \in H_{y}} \Delta^{-1}(x+t+y) \subseteq O_{S} \phi O_{F}$. Since $O_{S} \phi O_{F} \subseteq \Delta^{-1}\left(\Delta(\phi)+H_{S}+H_{F}\right)$ holds by construction, we are done.

Corollary 5.4. Let $K$ be a commutative semisimple Hausdorff topological ring such that $K^{\times}$is open in $K$ and $a \mapsto a^{-1}: K^{\times} \rightarrow K^{\times}$is continuous. Let $F$ be $a$ dense topological subfield of $K$, let $S$ be an open subring of $K$, and let $R=S \cap F$. Let $(A, \sigma, u, \Lambda)$ be a unitary $R$-algebra such that $A$ is flat as an $R$-module and $\operatorname{dim}_{F}\left(A_{F}\right)<\infty$, let $(P,[f]) \in \mathrm{UQ}^{u, \Lambda}(A, \sigma)$, and let $\mathcal{I}=\mathcal{I}\left(P_{K}\right)$ and $\Delta=\Delta_{\mathcal{I},\left[f_{K}\right]}$. Then

$$
\left|\operatorname{gen}_{S, F}(P,[f])\right|=\frac{2^{|\mathcal{I}|}}{\left|\Delta\left(O\left(\left[f_{F}\right]\right)\right)+\Delta\left(O\left(\left[f_{S}\right]\right)\right)\right|} .
$$

In addition, for all $\left(P^{\prime},\left[f^{\prime}\right]\right) \in \operatorname{gen}_{S, F}(P,[f])$, we have $P^{\prime} \cong P$.

Proof. The ring $A_{K}$ is artinian and hence semiperfect. Thus, by Theorem 3.1 and the proof of Theorem 3.4. $\Delta$ is onto, namely, $\Delta\left(O\left(\left[f_{K}\right]\right)\right)=(\mathbb{Z} / 2 \mathbb{Z})^{\mathcal{I}}$. If $\mathbb{F}_{2}$ is an epimorphic image of $K$, then necessarily $F=\mathbb{F}_{2}$ (because $F$ is a field). Since $K$ is Hausdorff and $F$ is dense in $K$, we must have $R=S=F=K=\mathbb{F}_{2}$ and the corollary becomes a triviality. We may therefore assume that $\mathbb{F}_{2}$ is not an epimorphic image of $K$. The corollary then follows from Theorem [5.1 provided we verify conditions (1)-(5). Indeed, conditions (1) and (5) hold by assumption and conditions (2) and (3) hold since $F$ is a field and $\operatorname{dim}_{F} A_{F}<\infty$. That $A$ is flat as an $R$-module holds by assumption. To see that $K$ is flat over $R$, observe that $K$ is flat over $F_{0}$, the fraction field of $R$, and $F_{0}$ is flat over $R$. This proves condition (4). (When $K$ is non-discrete, we actually have $F_{0}=F$ : Since $S$ is open in $K, R=S \cap F$ is open in $F_{0}$. Thus, $F_{0}$ is open in $F$. Let $x \in F$. Then there is a neighborhood $U \subseteq F_{0}$ of 0 such that $(x+U)(0+U) \subseteq 0+F_{0}$. Since $F$ is non-discrete, $U \neq\{0\}$, so there are $a, b \in F_{0}^{\times}$such that $(x+a) b \in F_{0}$. But this means $x \in F_{0}$.)

The following example shows that in general $\operatorname{gen}_{S, F}(P,[f])$ can be of size $2^{|\mathcal{I}|}$ for arbitrarily large $\mathcal{I}=\mathcal{I}\left(P_{K}\right)$.

Example 5.5. We use the notation of Example 5.2 and assume that $\operatorname{char} F \neq 2$ and $\mathbb{F}_{2}$ is not an epimorphic image of $R$. Let $\mathfrak{p}_{1}, \ldots, \mathfrak{p}_{t}$ be the maximal ideals of $R$, and for convenience, set $S_{i}=\hat{R}_{\mathfrak{p}_{i}}$ and $K_{i}=\hat{F}_{\mathfrak{p}_{i}}$. Then $K=\prod_{i} K_{i}$ and $S=\prod_{i} S_{i}$.

Fix some $u, v \in R^{\times}$and $0 \neq \pi \in \operatorname{Jac}(R)$. For a commutative $R$-algebra $T$, let $(u, v)_{T}$ denote the quaternion algebra with center $T$ determined by $u$ and $v$, namely, $T\left\langle x, y \mid x^{2}=u, y^{2}=v, x y=-y x\right\rangle$. Let $\sigma=\sigma_{T}$ be the involution given by

$$
(a+b x+c y+d x y)^{\sigma}=a+b x+c y-d x y \quad \forall a, b, c, d \in T .
$$

Define

$$
A=R+\pi R x+\pi R y+\pi R x y \subseteq(u, v)_{R}
$$

and set $\Lambda=\Lambda^{\min }(1)=\left\{a-a^{\sigma} \mid a \in A\right\}$. Then $(A, \sigma, 1, \Lambda)$ is a unitary $R$-algebra. Assume further that $(u, v)_{F}$ is a division algebra and $(u, v)_{K_{i}}$, which is a centralsimple $K_{i}$-algebra, splits for all $i$. We claim that for all $(P,[f]) \in \mathrm{UQ}^{u, \Lambda}(A, \sigma)$ with $P \neq 0$, we have $\left|\mathcal{I}\left(P_{K}\right)\right|=t$ and $\operatorname{gen}_{S, F}(P,[f])=2^{\left|\mathcal{I}\left(P_{K}\right)\right|}=2^{t}$. 
Indeed, we clearly have

$$
\left(A_{K}, \sigma_{K}, 1, \Lambda_{K}\right) \cong \prod_{i}\left((u, v)_{K_{i}}, \sigma_{K_{i}}, 1, \Lambda_{K_{i}}\right),
$$

and each of the factors is split-orthogonal by assumption. Furthermore, since $P_{F} \neq 0, P_{K_{i}} \neq 0$ for all $1 \leq i \leq t$. It follows that we can identify $\mathcal{I}\left(P_{K}\right)$ with $\{1, \ldots, t\}$. Denote by $\Delta_{i}$ the map $\Delta_{\left[f_{K_{i}}\right]}: O\left(\left[f_{K_{i}}\right]\right) \rightarrow \mathbb{Z} / 2 \mathbb{Z}$. By Corollary 5.4 and the definition of $\Delta_{\mathcal{I}\left(P_{K}\right),\left[f_{K}\right]}$, it is enough to show that $\Delta_{i}\left(O\left(\left[f_{F}\right]\right)\right)=0$ and $\Delta_{i}\left(O\left(\left[f_{S_{i}}\right]\right)\right)=0$ for all $1 \leq i \leq t$.

Let us first show that $\Delta_{i}\left(O\left(\left[f_{S_{i}}\right]\right)\right)=0$. We have $A_{S_{i}}=S_{i}+\pi S_{i} x+\pi S_{j} y+\pi S_{i} x y$. It is easy to check that $\pi \cdot(u, v)_{S_{i}} \subseteq \operatorname{Jac}\left(A_{S_{i}}\right)$. This means $A_{S_{i}} / \operatorname{Jac}\left(A_{S_{i}}\right)$ is isomorphic to an epimorphic image of $S_{i} / \pi S_{i}$, hence $A_{S_{i}}$ is local and $A_{S_{i}} / \operatorname{Jac}\left(S_{i}\right)$ is isomorphic to $R / \mathfrak{p}_{i}$. Thus, $O\left(\left[f_{S_{i}}\right]\right)$ is generated by reflections (Theorem 3.1 ] $\mathbb{F}_{2}$ is not an epimorphic image of $R$ ), so it is enough to prove that $\Delta_{i}\left(\phi_{K_{i}}\right)=0$ for every reflection $\phi$ of $\left[f_{S_{i}}\right]$. Indeed, in this case, $\phi_{K_{i}}$ is a reflection of $\left[f_{K_{i}}\right]$ and hence $\Delta_{i}\left(\phi_{K_{i}}\right)=\operatorname{deg} A_{K_{i}}+2 \mathbb{Z}=0$ by [17, Pr. 5.2]. This argument also shows that $\Delta_{i}\left(O\left(P_{F},\left[f_{F}\right]\right)\right)=0$ (because $A_{F}=(u, v)_{F}$ is a division ring, and hence local). Alternatively, $\Delta_{i}\left(O\left(P_{F},\left[f_{F}\right]\right)\right)=0$ follows from [17, Pr. 5.1].

Explicit choices of $R, u, v, \pi$ satisfying all previous conditions are given as follows: Take $R$ to be $\mathbb{Z}$ localized at the multiplicative set $\mathbb{Z} \backslash \bigcup_{i=1}^{t} \mathbb{Z} p_{i}$ where $p_{1}, \ldots, p_{t}$ are distinct odd prime numbers, let $u=v=-1$, and take $\pi=p_{1} \cdots p_{t}$.

Remark 5.6. In general, $\operatorname{gen}_{F}(P,[f])$ and $\operatorname{gen}_{S}(P,[f])$ can be infinite even when $R, S, F, K$ are as in Example 5.2. For example, take $K=\mathbb{C}(s)((t))$ with the $t$-adic topology, $S=\mathbb{C}(s)[[t]], F=K(s)(t)$ and $R=S \cap F$. Define $A=\left[\begin{array}{c}R \\ R\end{array} t^{2} R\right]$ and $\sigma: A \rightarrow A$ by $\left[\begin{array}{cc}x & t^{2} y \\ z & w\end{array}\right]^{\sigma}=\left[\begin{array}{cc}x & t^{2} z \\ y & w\end{array}\right](x, y, z, w \in R)$, and let $\Lambda=\Lambda^{\min }(1)$. Then $(A, \sigma, 1, \Lambda)$ is a unitary $R$-algebra with $A_{S}=\left[\begin{array}{c}S \\ t_{S}^{2} S\end{array}\right]$ and $A_{F}=\mathrm{M}_{2}(F)$. The assumptions of Theorem 5.1 are easily seen to hold.

For every $a \in A$ with $a^{\sigma}=a$, define a quadratic space $\left(A,\left[f_{a}\right]\right)$ by

$$
\tilde{f}_{a}(x, y)=x^{\sigma} a y \text {. }
$$

We claim that both $\operatorname{gen}_{F}\left(A,\left[f_{1}\right]\right)$ and $\operatorname{gen}_{S}\left(A,\left[f_{1}\right]\right)$ consist of infinitely many isomorphism classes.

Indeed, it is easy to check that $\left[f_{a}\right] \cong\left[f_{b}\right]$ if and only if there exists $x \in A^{\times}$such that $x^{\sigma} a x=b$. Since for all $\alpha \in \mathbb{C}$, we have

$$
b(\alpha):=\left[\begin{array}{cc}
1+\alpha^{2} s^{2} & 0 \\
0 & 1+\alpha^{2} s^{2}
\end{array}\right]=\left[\begin{array}{cc}
1 & -\alpha t s \\
\alpha t^{-1} s & 1
\end{array}\right]^{\sigma} \cdot 1_{A} \cdot\left[\begin{array}{cc}
1 & -\alpha t s \\
\alpha t^{-1} s & 1
\end{array}\right],
$$

it follows that $\left(A,\left[f_{b(\alpha)}\right]\right) \in \operatorname{gen}_{F}\left(A,\left[f_{1}\right]\right)$ for all $\alpha \in \mathbb{C}$. However, the forms $\left\{\left[f_{b(\alpha)}\right] \mid \operatorname{Im}(\alpha)>0\right\}$ are pairwise non-isomorphic over $(A, \sigma, 1, \Lambda)$, as can be easily checked by working modulo $\operatorname{Jac}(A)=\left[\begin{array}{cc}t R & t^{2} R \\ R & t R\end{array}\right]$.

Next, observe that for all $\alpha \in \mathbb{C}, 1+\alpha t$ has a square root in $S$ (substitute $\alpha t$ in the Taylor expansion of $\sqrt{1+x}$ ), but not in $R$. Since

$$
c(\alpha):=\left[\begin{array}{cc}
1+\alpha t & 0 \\
0 & 1
\end{array}\right]=\left[\begin{array}{cc}
\sqrt{1+\alpha t} & 0 \\
0 & 1
\end{array}\right]^{\sigma} \cdot 1_{A} \cdot\left[\begin{array}{cc}
\sqrt{1+\alpha t} & 0 \\
0 & 1
\end{array}\right],
$$

we have $\left(A,\left[f_{c(\alpha)}\right]\right) \in \operatorname{gen}_{S}\left(A,\left[f_{1}\right]\right)$ for all $\alpha \in \mathbb{C}$. The forms $\left\{\left[f_{c(\alpha)}\right]\right\}_{\alpha \in \mathbb{C}}$ are pairwise non-isomorphic because $\left[f_{c(\alpha)}\right] \cong\left[f_{c\left(\alpha^{\prime}\right)}\right] \operatorname{implies} \operatorname{det}(c(\alpha)) \in \operatorname{det}\left(c\left(\alpha^{\prime}\right)\right)\left(F^{\times}\right)^{2}$ and this is possible only if $\alpha=\alpha^{\prime}$. 
We remark that $\left|\operatorname{gen}_{S, F}(P,[f])\right|=1$ for all $(P,[f]) \in \mathrm{UQ}^{1, \Lambda}(A, \sigma)$ by Theorem 6.2 below. Replacing $\mathbb{C}$ with a larger field allows one to construct similar examples in which the $F$-genus and $S$-genus are of arbitrarily large cardinalities.

\section{Genus of SIZE 1}

This section gives several sufficient conditions guaranteeing that the $\{S, F\}$-genus of Theorem 5.1 and Corollary 5.4 has size 1 for all quadratic spaces. In 6A, we assume the general setting of Theorem 5.1 and in $6 \mathrm{~B}$ we specialize to orders over semilocal principal ideal domains.

6A. General criteria. We assume the setting of Theorem 5.1. That is, $K$ is a semilocal topological ring, $F$ and $S$ are subrings of $K, R=S \cap F,(A, \sigma, u, \Lambda)$ is a unitary $R$-algebra, $(P,[f]) \in \mathrm{UQ}^{u, \Lambda}(A, \sigma)$, and all assumptions of Theorem 5.1 hold. We further write $(B, \tau, v, \Gamma)=\mathcal{R}_{K / R}(A, \sigma, u, \Lambda), \bar{B}=B / \operatorname{Jac}(B)$, and $(\bar{B}, \bar{\tau}, \bar{v}, \bar{\Gamma})=$ $\prod_{i=1}^{t}\left(B_{i}, \tau_{i}, v_{i}, \Gamma_{i}\right)$ where each factor is a simple unitary ring (see 1D).

We note that since $A$ is flat over $R$, the map $A \rightarrow A_{F}$ is injective (because the inclusion map $R \rightarrow F$ is injective). The maps $A \rightarrow A_{S}, A \rightarrow A_{K}, A_{S} \rightarrow A_{K}$, $A_{F} \rightarrow A_{K}$ are likewise injective.

We begin by observing that when $\mathcal{I}\left(A_{K}\right)=\emptyset$, we must have $\left|\operatorname{gen}_{S, F}(P,[f])\right|=1$ by Theorem 5.1. This happens, for example, when the involutions $\tau_{1}, \ldots, \tau_{t}$ are all of the second kind, in which case we say that $\tau=\sigma_{K}$ is essentially of the second kind. This condition is equivalent to the existence of $a \in A_{K}$ such that $a+\operatorname{Jac}\left(A_{K}\right)$ is central in $\overline{A_{K}}:=A_{K} / \operatorname{Jac}\left(A_{K}\right)$ and $a-a^{\sigma_{K}} \in A_{K}^{\times}$; the easy proof is left to the reader. We record our conclusion in the following proposition.

Proposition 6.1. If $\sigma_{K}$ is essentially of the second kind, then $\left|\operatorname{gen}_{S, F}(P,[f])\right|=1$ for all $(P,[f]) \in \mathrm{UQ}^{u, \Lambda}(A, \sigma)$.

The next theorem is a general criterion that can be applied to particular examples. It will be used in the proofs of Theorems 6.7 and 7.4 below.

Recall that two idempotents $e, e^{\prime}$ in a ring $W$ are called equivalent, denoted $e \approx e^{\prime}$, if $e W \cong e^{\prime} W$ as right $W$-modules. This is equivalent to the existence of $x \in e^{\prime} W e$ and $y \in e W e^{\prime}$ such that $y x=e$ and $x y=e^{\prime}$. Recall also that an idempotent $e$ is called primitive if $e W e$ does not contain idempotents beside 0 and $e$. When $W$ is semiperfect (see $3 \mathrm{~A}$ ), the latter is equivalent to $e W e$ being local. Furthermore, in this case, there are finitely many equivalence classes of primitive idempotents. More precisely, if $\bar{W}:=W / \operatorname{Jac}(W) \cong \prod_{i=1}^{t} \mathrm{M}_{n_{i}}\left(D_{i}\right)$ with each $D_{i}$ a division ring, and if $\varepsilon_{i}$ is a primitive idempotent in $\mathrm{M}_{n_{i}}\left(D_{i}\right)$ (viewed as an idempotent in $\bar{W})$, then any family of idempotents $e_{1}, \ldots, e_{t} \in W$ with $\varepsilon_{i}=e_{i}+\operatorname{Jac}(W)$ is a family of representatives for the equivalence classes.

Theorem 6.2. Assume $A_{S}$ is semilocal, $A_{K}$ is semiperfect, and one of the following equivalent conditions hold:

(1) For every primitive idempotent $e \in A_{K}$, there is an idempotent $e^{\prime} \in A_{S}$ with $e \approx e^{\prime}$.

(2) There are idempotents $e_{1}, \ldots, e_{s} \in A_{S}$ such that $A_{K}=\sum_{j} A_{K} e_{j} A_{K}$ and $e_{j} A_{K} e_{j}$ is local for all $j$.

Then $\left|\operatorname{gen}_{S, F}(P,[f])\right|=1$ for all $(P,[f]) \in \mathrm{UQ}^{u, \Lambda}(A, \sigma)$. 
Proof. Recall that $B=A_{K}$. We first prove that conditions (1) and (2) are equivalent. Suppose (1) holds. We can write $1_{B}=\sum_{j=1}^{s} e_{j}$ where $e_{1}, \ldots, e_{s}$ are primitive idempotents in $B$. We clearly have $B=\sum_{j} B e_{j} B$ and $e_{j} B e_{j}$ is local for all $j$. For each $j$, choose $e_{j}^{\prime} \in A_{S}$ such that $e_{j}^{\prime} \approx e_{j}$. It is easy to check that $e_{j} \approx e_{j}^{\prime}$ implies $B e_{j}^{\prime} B=B e_{j} B$ and $e_{j}^{\prime} B e_{j}^{\prime} \cong e_{j} B e_{j}$. Thus, the idempotents $e_{1}^{\prime}, \ldots, e_{s}^{\prime}$ satisfy condition (2). Conversely, assume (2) holds and let $e \in B$ be a primitive idempotent. The assumption $\sum_{j} B e_{j} B=B$ is easily seen to imply that there is a projection $\bigoplus_{j}\left(e_{j} B\right)^{n_{j}} \rightarrow B$ for some $n_{1}, \ldots, n_{s} \in \mathbb{N}$, and hence there is a projection $\bigoplus_{j}\left(e_{j} B\right)^{n_{j}} \rightarrow e B$. Let $J=\operatorname{Jac}(B)$. Then there is some $j$ for which there is a homomorphism $p: e_{j} B \rightarrow e B$ whose image is not contained in $e J$. Since $e B e$ is local, $\bar{e}:=e+J$ is primitive in $\bar{B}=B / J$, and since $\bar{B}$ is semisimple, $e B / e J \cong \bar{e}(B / J)$ is simple. Thus, $\operatorname{im}(p)+e J=e B$ (because $\operatorname{im}(p) \nsubseteq e J)$, and by Nakayama's Lemma we get $\operatorname{im}(p)=e B$. Since $e B$ is projective, this means that $e B$ is isomorphic to a summand of $e_{j} B$. But $\operatorname{End}_{B}\left(e_{j} B\right) \cong e_{j} B e_{j}$ is local, so the only summands of $e_{j} B$ are 0 and $e_{j} B$. Thus, $e B \cong e_{j} B$ and we have $e \approx e_{j} \in A_{S}$.

We now prove that condition (1) implies $\left|\operatorname{gen}_{S, F}(P,[f])\right|=1$. We may assume $P \neq 0$. Let $(Q,[g])=\left(P_{K},\left[f_{K}\right]\right)$. As in $\left.3 \mathrm{~A}\right](Q, g)$ induces quadratic spaces $\left(Q_{i},\left[g_{i}\right]\right) \in \mathrm{UQ}^{v_{i}, \Gamma_{i}}\left(B_{i}, \tau_{i}\right)$ for all $1 \leq i \leq t$. Let $\mathcal{I}=\mathcal{I}\left(P_{K}\right)$. By Theorem [5.1, it is enough to prove that $\Delta_{\mathcal{I},[g]}\left(O\left(P_{S},\left[f_{S}\right]\right)\right)=(\mathbb{Z} / 2 \mathbb{Z})^{\mathcal{I}}$. By Proposition 3.3 , we may apply transfer to assume that $P$ is free, and hence so is $Q$.

We proceed by recalling some results and notions from [17]: Suppose that we are given an idempotent $e \in B$, an element $y \in Q e$, and $c \in \hat{g}(y) \cap e^{\sigma} B e=$ $(\tilde{g}(y, y)+\Gamma) \cap e^{\sigma} B e$ such that multiplication on the left by $c$ induces an isomorphism $e B \rightarrow e^{\sigma} B$. The inverse of $x \mapsto c x: e B \rightarrow e^{\sigma} B$ is given by left multiplication with a unique element of $e B e^{\sigma}$, which we denote by $c^{\circ}$. In this setting, the map $s_{y, e, c}: Q \rightarrow Q$ given by

$$
s_{y, e, c}(x)=x-y c^{\circ} \tilde{h}_{g}(y, x) \quad \forall x \in Q
$$

is called an e-reflection of $[g]$. It is always an isometry. If $e^{\prime} \in A$ is another idempotent with $e \approx e^{\prime}$, then every $e$-reflection is an $e^{\prime}$-reflection (for different $y$ and $c$ ). Furthermore, if $\phi$ is an $e$-reflection, then the induced isometry $\bar{\phi} \in O(\bar{Q},[\bar{g}])$ is an $\bar{e}$-reflection, and every $\bar{e}$-reflection is obtained in this way. Finally, we record that $\bar{e}$-reflections exist when $\bar{e}$ is primitive and $\overline{P e} \neq 0$. See [17, $\S 3, \S 5.2]$ for proofs.

We now return to the proof: Let $i \in \mathcal{I}$. Then $B_{i} \cong \mathrm{M}_{n}(L)$ for some field $L$ and $n \in \mathbb{N}$. Let $\varepsilon \in \mathrm{M}_{n}(L) \cong B_{i}$ denote the idempotent matrix whose $(1,1)$-entry is 1 and its other entries are 0 . Since $B$ is semiperfect, there is an idempotent $e \in B$ whose image in $\bar{B}$ is $\varepsilon$. The idempotent $e$ is primitive, so by condition (1) there is an idempotent $e^{\prime} \in A_{S}$ with $e^{\prime} \approx e$.

Suppose $\left[f_{S}\right]$ has an $e^{\prime}$-reflection $\phi$. Then $\phi_{K}$ is an $e^{\prime}$-reflection of $[g]=\left[f_{K}\right]$, and since $e \approx e^{\prime}, \phi_{K}$ is also an $e$-reflection. This implies that the induced isometry $\phi_{i} \in O\left(\left[g_{i}\right]\right)$ is an $\varepsilon$-reflection, while $\phi_{j}=\operatorname{id}_{Q_{j}}$ for all $j \in \mathcal{I} \backslash\{i\}$. By [17, Pr. 5.2], $\Delta_{\left[g_{i}\right]}\left(\phi_{i}\right)=1+2 \mathbb{Z}$, so $\Delta_{\mathcal{I},[g]}(\phi)=\left(\delta_{i j}+2 \mathbb{Z}\right)_{j \in \mathcal{I}}$ where $\delta_{i j}=1$ if $i=j$ and $\delta_{i j}=0$ otherwise.

We now claim that $\left[f_{S}\right]$ has an $e^{\prime}$-reflection and hence that $\left(\delta_{i j}+2 \mathbb{Z}\right)_{j \in \mathcal{I}} \in$ $\Delta_{\mathcal{I},[g]}\left(O\left(\left[f_{S}\right]\right)\right)$. Let ${ }^{`}$ denote reduction modulo $\operatorname{Jac}\left(A_{S}\right)$ (whenever it makes sense). Then it is enough to prove that $\left(\check{P}_{S},\left[\check{f}_{S}\right]\right)$ has an $\check{e}^{\prime}$-reflection. Write $\check{e}^{\prime}$ as a sum of orthogonal primitive idempotents in $\breve{A}_{S}, \check{e}^{\prime}=\sum_{j} e_{j}$. It is enough to prove that $\left(\check{P}_{S},\left[\check{f}_{S}\right]\right)$ has an $e_{j}$-reflection for all $j$ because their product will be an $\check{e}^{\prime}$-reflection 
by [17, Lm. 3.4]. Since $P$ is free and non-zero, $\check{P}_{S}$ is free and non-zero, and hence $\check{P}_{S} e_{j} \neq 0$ for all $j$. This implies that $e_{j}$-reflections exist, as required.

By letting $i$ range over $\mathcal{I}$, we have now shown that $\Delta_{\mathcal{I},[g]}\left(O\left(\left[f_{S}\right]\right)\right)$ contains $\left\{\left(\delta_{i j}+2 \mathbb{Z}\right)_{j \in \mathcal{I}}\right\}_{i \in \mathcal{I}}$. This set spans $(\mathbb{Z} / 2 \mathbb{Z})^{\mathcal{I}}$ as a group, so we are done.

Example 6.3. Let $R, S=\prod_{i=1}^{t} S_{i}, F, K=\prod_{i=1}^{t} K_{i}$ be as in Example 5.5, Let $\mathfrak{a}$ be a non-zero ideal of $R$, let

$$
A=\left[\begin{array}{cc}
R & \mathfrak{a} \\
\mathfrak{a} & R
\end{array}\right]
$$

let $\sigma$ be the transpose involution and let $\Lambda=\left\{a-a^{\sigma} \mid a \in A\right\}$. Then $(A, \sigma, 1, \Lambda)$ is a unitary ring and

$$
B=A_{K}=\prod_{i=1}^{t} \mathrm{M}_{2}\left(K_{i}\right), \quad A_{S}=\prod_{i=1}^{t}\left[\begin{array}{cc}
S_{i} & \mathfrak{a} S_{i} \\
\mathfrak{a} S_{i} & S_{i}
\end{array}\right] .
$$

Let $e_{i}$ denote the matrix unit $e_{11}$ of $\mathrm{M}_{2}\left(K_{i}\right)$, viewed as an element of $A_{S}$. It is easy to see that $e_{i} B e_{i}$ is local for all $i$ and $\sum_{i=1}^{t} B e_{i} B=B$. Thus, by Theorem 6.2, $\left|\operatorname{gen}_{S, F}(P,[f])\right|=1$ for all $(P,[f]) \in \mathrm{UQ}^{u, \Lambda}(A, \sigma)$. (Despite this, as in Example 5.5. we have $\mathcal{I}\left(P_{K}\right)=\{1, \ldots, t\}$ for all $0 \neq P \in \mathscr{P}(A)$.)

Example 6.4. Let $R, S, F, K$ and $(A, \sigma, u, \Lambda)$ be as in Example 5.5. Then $A_{K}$ is semiperfect (because it is artinian) and $A_{S}$ is semilocal, but conditions (1) and (2) of Theorem 6.2 do not hold for $A$. Indeed, if this was not so, then the computation of $\left|\operatorname{gen}_{S, F}(P,[f])\right|$ in Example 5.5 would contradict the conclusion of Theorem 6.2.

The failure of (1) and (2) can also be checked directly: In the notation of Example 5.5. the ring $A_{S_{i}}$ is local for all $1 \leq i \leq t$, hence the only non-zero idempotent in $A_{S_{i}}$ is its unity, denoted $e_{i}$. Since $A_{S}=\prod_{i=1}^{t} A_{S_{i}}$, any idempotent $e \in A_{S}$ can be written as $\sum_{i \in I} e_{i}$ for $I \subseteq\{1, \ldots, t\}$, in which case $e A_{K} e \cong \bigoplus_{i \in I} A_{K_{i}}=$ $\prod_{i \in I} \mathrm{M}_{2}\left(K_{i}\right)$. It follows that $e A_{K} e$ is not local for any idempotent $0 \neq e \in A_{S}$, so (2) is false. The failure of (1) can be checked by similar means.

6B. Orders. Assume henceforth that $R$ is a semilocal PID with maximal ideals $\mathfrak{p}_{1}, \ldots, \mathfrak{p}_{t}$ and fraction field $F$. As in Example [5.5, we set $S=\prod_{i=1}^{t} \hat{R}_{\mathfrak{p}_{i}}, K=$ $\prod_{i=1}^{t} \hat{F}_{\mathfrak{p}_{i}}$ and $\hat{R}_{0}=F$. We further assume that $R \neq F$.

Recall that an $R$-order in an $F$-algebra $E$ is an $R$-subalgebra $A \subseteq E$ which is finitely generated as an $R$-module and contains an $F$-basis of $E$. Since $R$ is a PID, an $R$-algebra $A$ is an $R$-order in some $F$-algebra (necessarily isomorphic to $A_{F}$ ) if and only if $A$ is a finitely generated free $R$-module.

An $R$-order $A$ is called hereditary if its one-sided ideals are projective as $A$ modules. For example, $R$ itself is hereditary because it is a PID. The structure of hereditary $R$-orders is well-understood and we refer the reader to [31, Ch. 9] for further details.

Example 6.5. (i) An $R$-order $A$ is maximal if it is not properly contained in any $R$-order inside $A_{F}$. It is well-known that maximal $R$-orders are hereditary [21, Cor. 1.6.0].

(ii) Certain weak crossed products over $R$ (i.e. crossed products defined using 2 -cocycles taking non-invertible values) are hereditary (or semihereditary); see 22 and related papers. 
(iii) Let $\pi \in R$ be a prime element. Then

$$
A=\left[\begin{array}{cc}
R & \pi R \\
R & R
\end{array}\right]
$$

is a non-maximal hereditary order. This can be checked directly, or by using the results in [31, Ch. 9].

(iv) The $R$-order of Example 5.6 is not hereditary. The $R$-order $A$ of Example 6.3 is not hereditary unless $\mathfrak{a}=R$.

We shall make extensive usage of the following theorem.

Theorem 6.6. Let $A$ be an R-order. Then $A$ is hereditary if and only if $A_{\hat{R}_{\mathfrak{p}}}$ is hereditary for all $0 \neq \mathfrak{p} \in \operatorname{Spec} R$ (i.e. if $A_{S}$ is hereditary). In this case, $A_{F}$ and $A_{\hat{F}_{\mathfrak{p}}}$ are semisimple for all $0 \neq \mathfrak{p} \in \operatorname{Spec} R$.

Proof. By [2, p. 8], the order $A$ is hereditary if and only if $A_{R_{\mathfrak{p}}}$ is hereditary for all $0 \neq \mathfrak{p} \in \operatorname{Spec} R$. We may therefore assume $R$ is a DVR. Write $\hat{R}$ and $\hat{F}$ for the completions of $R$ and $F$. Recall that the order $A$ is called extremal if there is no order $A \subsetneq A^{\prime} \subseteq A_{F}$ with $\operatorname{Jac}(A) \subseteq \operatorname{Jac}\left(A^{\prime}\right)$. By [21, Th. 1.6], the hereditary orders are precisely the extremal orders. Observe that tensoring with $\hat{R}$ induces a bijection between the full $R$-lattices in $A_{F}$ (i.e. finitely generated $R$-modules containing an $F$-basis of $A_{F}$ ) and the full $\hat{R}$-lattices in $A_{\hat{F}}$; see [31, Th. 5.2(ii)], for instance. This bijection clearly takes $R$-orders to $\hat{R}$-orders, maximal left ideals to maximal left ideals, and hence the Jacobson radical of an $R$-order $A^{\prime} \subseteq A_{F}$ to the Jacobson radical of $A_{\hat{R}}^{\prime}$. As a result, $A$ is extremal if and only if $A_{\hat{R}}$ is extremal. This proves the first statement of the theorem 1

That $A_{F}$ and $A_{\hat{F}_{\mathrm{p}}}$ are semisimple follows from [21, Th. 1.7.1], stating that any finite-dimensional $F$-algebra with a hereditary $R$-order is semisimple.

The following theorem is a special case of Theorem 6.2

Theorem 6.7. Let $(A, \sigma, u, \Lambda)$ be a unitary $R$-algebra such that $A$ is a hereditary $R$-order. Then $\left|\operatorname{gen}_{\{F, S\}}(P,[f])\right|=1$ for all $(P,[f]) \in \mathrm{UQ}^{u, \Lambda}(A, \sigma)$.

Proof. It is clear that $A_{K}$ is semiperfect, $A_{S}$ is semilocal, and conditions (1)-(5) of Theorem 5.1 are satisfied. As in Corollary 5.4, we can ignore condition (0) of Theorem 5.1] It is therefore enough to verify condition (1) of Theorem 6.2.

By Theorem 6.6, $A_{S}$ is hereditary. Let $e$ be a primitive idempotent in $A_{K}$, and let $I=A_{S} \cap e A_{K}$. Define a right $A_{S}$-module homomorphism $\psi: A_{S} / I \rightarrow(1-e) A_{K}$ by $\psi(a+I)=(1-e) x$ (this is well defined because $(1-e) I=0)$. We claim that $\psi$ is injective. Indeed, if $\psi(a+I)=0$, then $(1-e) a=0$ and hence $a=e a$, which means $a \in I$. Since $A_{S} / I$ is a cyclic $A_{S}$-module and $K=F \cdot S$, the image of $\psi$ is contained in $t A_{S}$ for some $t \in F$. Therefore, $A_{S} / I$ is isomorphic to a right ideal of $A_{S}$. Since $A_{S}$ is hereditary, $A_{S} / I$ is projective, so the exact sequence $0 \rightarrow I \rightarrow A_{S} \rightarrow A_{S} / I \rightarrow 0$ splits. We therefore have $A_{S}=I \oplus J$ where $J$ is some right ideal of $A_{S}$. Write $1=e^{\prime}+a$ with $e^{\prime} \in I$ and $a \in J$. It is easy to check that $e^{\prime}$ is an idempotent satisfying $e^{\prime} A_{S}=I$ and $e^{\prime} A_{K}=e A_{K}$, so $e^{\prime} \approx e$.

\footnotetext{
${ }^{1}$ This statement also follows from [31, Th. 3.30]. However, the proof there seems to have a gap because it assumes that all $A_{\hat{R}_{\mathfrak{p}}}$-modules are obtained from $A$-modules via scalar extension, which is false in general.
} 
Corollary 6.8. Keep the assumptions of Theorem 6.7 and let $(P,[f]),\left(P^{\prime},\left[f^{\prime}\right]\right) \in$ $\mathrm{UQ}^{u, \Lambda}(A, \sigma)$. For all $\mathfrak{p} \in \operatorname{Spec} R$, let $k(\mathfrak{p})$ denote the fraction field of $R / \mathfrak{p}$. Then $(P,[f]) \cong\left(P^{\prime},\left[f^{\prime}\right]\right)$ if and only if $\left(P_{k(\mathfrak{p})},\left[f_{k(\mathfrak{p})}\right]\right) \cong\left(P_{k(\mathfrak{p})}^{\prime},\left[f_{k(\mathfrak{p})}^{\prime}\right]\right)$ for all $\mathfrak{p} \in \operatorname{Spec} R$ (including $\mathfrak{p}=0$ ).

Proof. We have $A \in \mathscr{P}(R)$ by assumption. Thus, as noted in Example 5.2. $\operatorname{gen}_{S, F}(P,[f])=\operatorname{gen}_{\{k(\mathfrak{p}) \mid \mathfrak{p} \in \operatorname{Spec} R\}}(P,[f])$. The corollary therefore follows from Theorem 6.7

Remark 6.9. Theorem 6.7 actually holds in the general setting of Theorem 5.1 if one assumes that $A_{K}$ is semiperfect, $A_{S}$ is semilocal and semihereditary, and $K=U^{-1} S$ where $U=K^{\times} \cap S$. The proof is essentially the same.

\section{FURThER QUADRATIC OBJECTS}

We keep the notation of 6B. In this final section, we extend Theorem 5.1 to systems of sesquilinear forms and Theorem 6.7 to non-unimodular hermitian forms. This will be done using results from [5], 10] and [7]. As an application, we prove that Witt's Cancellation Theorem and Springer's Theorem hold for hermitian forms over hereditary orders.

In contrast to the previous sections, the results of this section require that $2 \in R^{\times}$.

7A. Systems of sesquilinear forms. Let $A$ be an $R$-order (see 6B) and let $\left\{\sigma_{i}\right\}_{i \in I}$ be a family of $R$-involutions on $A$. Then each involution $\sigma_{i}$ induces a duality $*_{i}: \mathscr{P}(A) \rightarrow \mathscr{P}(A)$ as in $1 \mathrm{~A}$. A system of sesquilinear forms over $\left(A,\left\{\sigma_{i}\right\}_{i \in I}\right)$ is a pair $\left(P,\left\{f_{i}\right\}_{i \in I}\right)$ such that $\left(P, f_{i}\right)$ is a sesquilinear form over $\left(A, \sigma_{i}\right)$ for all $i \in I$. If $\left(P^{\prime},\left\{f_{i}^{\prime}\right\}_{i \in I}\right)$ is another system, then an isometry from $\left(P,\left\{f_{i}\right\}\right)$ to $\left(P^{\prime},\left\{f_{i}^{\prime}\right\}\right)$ is an isomorphism of $A$-modules $\phi: P \rightarrow P^{\prime}$ such that $f_{i}=\phi^{*_{i}} f_{i}^{\prime} \phi$ for all $i \in I$.

The notion of genus naturally extends to systems of sesquilinear forms over $\left(A,\left\{\sigma_{i}\right\}_{i \in I}\right)$. For brevity, we let

$$
\operatorname{gen}\left(P,\left\{f_{i}\right\}\right)=\operatorname{gen}_{\left\{\hat{R}_{\mathfrak{p}} \mid \mathfrak{p} \in \operatorname{Spec} R\right\}}\left(P,\left\{f_{i}\right\}\right),
$$

where $\hat{R}_{0}=F$.

Theorem 7.1. Assume $2 \in R^{\times}$. Then, in the previous setting, $\left|\operatorname{gen}\left(P,\left\{f_{i}\right\}\right)\right|$ is a finite power of 2 .

The proof uses the language of hermitian categories. Our notation will follow [7, $\S 2, \S 4]$ and we refer the reader to this source for definitions; see also [30, 35. Ch. 7], [24, Ch. II] or [3].

Proof. By [7, Th. 4.1], there is an isomorphism between the category of systems sesquilinear forms over $\left(A,\left\{\sigma_{i}\right\}\right)$ and the category of unimodular 1-hermitian forms over a certain hermitian category, which we denote by $\mathscr{D}$ (in 7 , this category is denoted by $\mathrm{A}_{2 I}(\mathscr{C})$ where $\mathscr{C}=\mathscr{P}(A)$ ). Furthermore, this isomorphism is compatible with flat scalar extension over $R$ ([7, Cr. 4.4] and the comment before it). It is therefore enough to prove the theorem for unimodular 1-hermitian forms over the hermitian category $\mathscr{D}$.

Let $(Q, g)$ and $\left(Q^{\prime}, g^{\prime}\right)$ be two 1-hermitian forms over $\mathscr{D}$ with the same genus. We claim that $Q \cong Q^{\prime}$. Indeed, by applying transfer in hermitian categories (see [7, $\S 2 \mathrm{C}, \S 2 \mathrm{E}])$, we may assume $(Q, g)$ and $\left(Q^{\prime}, g^{\prime}\right)$ are 1-hermitian forms over a ring with involution $\left(A^{\prime}, \sigma^{\prime}\right)$ where $A^{\prime}$ is the endomorphism ring of some object in $\mathscr{D}$. 
By the construction of $\mathscr{D}, A^{\prime}$ an $R$-subalgebra of $\operatorname{End}_{A}\left(P_{1}\right) \times \operatorname{End}_{A}\left(P_{2}\right)^{\text {op }}$ for some $P_{1}, P_{2} \in \mathscr{P}(A)$ (see [7, §4]), and hence an $R$-order. In addition, since the hermitian structure on $\mathscr{D}$ is $R$-linear, $\sigma^{\prime}$ is an $R$-involution. Now, since $2 \in R^{\times}, 1$-hermitian forms are equivalent to quadratic spaces over $\left(A^{\prime}, \sigma^{\prime}, 1, \Lambda^{\max }(1)\right)$ (Remark 1.1). Thus, by Corollary 5.4. $Q^{\prime} \cong Q$.

This implies that for any $\left(Q^{\prime}, g^{\prime}\right) \in \operatorname{gen}(Q, g)$, we have $\left.Q^{\prime} \in \mathscr{D}\right|_{Q}$ (see [7, §2C] for the definition). In particular, gen $(Q, g)$ is contained in the category of unimodular 1-hermitian forms over $\left.\mathscr{D}\right|_{Q}$. The transfer functor with respect to $(Q, g)$ induces an equivalence between this category and the category of unimodular 1-hermitian forms over $\left(A^{\prime \prime}, \sigma^{\prime \prime}\right)$, where $A^{\prime \prime}=\operatorname{End}_{\mathscr{D}}(Q)$ and $\sigma^{\prime \prime}$ is induced from $g$. As before, $\left(A^{\prime \prime}, \sigma^{\prime \prime}\right)$ is an $R$-order with an $R$-involution, so we are reduced to the case where $(Q, g)$ is a 1-hermitian form over an $R$-order with an $R$-involution. Since $2 \in R^{\times}$, we may apply Corollary 5.4 and conclude that $\operatorname{gen}(Q, g)$ is a finite power of 2 .

One can guarantee that $\left|\operatorname{gen}\left(P,\left\{f_{i}\right\}\right)\right|=1$ in case all the involutions $\left\{\sigma_{i}\right\}_{i \in I}$ restrict to a particular involution on $\operatorname{Cent}(A)$ which is of the second kind: Let $R^{\prime} / R$ be a Galois extension (of commutative rings) with Galois group $\{\mathrm{id}, \tau\}(\tau \neq \mathrm{id}$ ); see [1, Apx.] or [34 for the general definition. In our case, this is equivalent to $R^{\prime} \cong R\left[x \mid x^{2}=a x+b\right]$ for $a, b \in R$ satisfying $a^{2}+4 b \in R^{\times}$, in which case $\tau$ is the $R$-automorphism of $R^{\prime}$ sending $x$ to $a-x$.

Theorem 7.2. Keep the previous setting and assume in addition that $A$ is an $R^{\prime}$ algebra and $\left.\sigma_{i}\right|_{R^{\prime}}=\tau$ for all $i \in I$. Then $\left|\operatorname{gen}\left(P,\left\{f_{i}\right\}\right)\right|=1$ for every system of sesquilinear forms $\left(P,\left\{f_{i}\right\}\right)$ over $\left(A,\left\{\sigma_{i}\right\}\right)$.

Proof. Let $\left(A^{\prime \prime}, \sigma^{\prime \prime}\right)$ be the $R$-order with involution constructed in the last paragraph of the proof of Theorem 7.1. We claim that $\sigma_{K}^{\prime \prime}$ is essentially of the second kind (see 6A), in which case we are done by Proposition 6.1. By the construction of the hermitian category $\mathscr{D}, A^{\prime \prime}$ is an $R^{\prime}$-algebra and $\left.\sigma^{\prime \prime}\right|_{R^{\prime}}=\tau$ (see [7, $\left.\S 5 \mathrm{~F}\right]$ ). Since $R^{\prime} / R$ is Galois with Galois group $\{\mathrm{id}, \tau\}, R_{F}^{\prime} / F$ is Galois with Galois group $\left\{\mathrm{id}, \tau_{F}\right\}$. In this case, either $R_{F}^{\prime} \cong F \times F$ or $R_{F}^{\prime} / F$ is a 2-dimensional field extension. In either case, it is easy to see that there is $a \in R_{F}^{\prime} \subseteq \operatorname{Cent}\left(A_{K}\right)$ such that $a-a^{\tau_{F}} \in\left(R_{F}^{\prime}\right)^{\times}$, and hence $\sigma_{K}^{\prime \prime}$ is essentially of the second kind.

Remark 7.3. Theorem 7.1 also holds for systems of sesquilinear forms over $R$ categories (see [7, §2D, §4]) in which the Hom-sets are finitely generated projective $R$-modules. The proof is similar.

7B. Non-unimodular Hermitian forms. Let $(A, \sigma)$ be an $R$-order with an $R$ involution and let $u \in \operatorname{Cent}(A)$ be an element such that $u^{\sigma} u=1$. We extend the notion of genus to (not-necessarily unimodular) $u$-hermitian forms in the obvious way. As in 7A, for every $u$-hermitian form $(P, f)$ over $(A, \sigma)$, we set

$$
\operatorname{gen}(P, f)=\operatorname{gen}_{\left\{\hat{R}_{\mathfrak{p}} \mid \mathfrak{p} \in \operatorname{Spec} R\right\}}(P, f)
$$

for brevity. (Note that if $(P, f)$ is unimodular and $\left(P^{\prime}, f^{\prime}\right) \in \operatorname{gen}(P, f)$, then $f^{\prime}$ is also unimodular by virtue of Corollary 4.3 .)

Viewing $(P, f)$ as a system of sesquilinear forms (consisting of just one form), Theorem 7.1 implies that $|\operatorname{gen}(P, f)|$ is a finite power of 2 . We now strengthen this assertion by showing that $|\operatorname{gen}(P, f)|=1$ when $A$ is hereditary (see 6B).

Theorem 7.4. Keep the previous setting and assume $A$ is hereditary and $2 \in R^{\times}$. Then $|\operatorname{gen}(P, f)|=1$ for any u-hermitian form $(P, f)$ over $(A, \sigma)$. 
We first prove the following lemma. We denote by $\operatorname{Mor}(\mathscr{P}(A))$ the category of morphisms in $\mathscr{P}(A)$. Its objects are triples $(U, g, V)$ with $U, V \in \mathscr{P}(A)$ and $g \in \operatorname{Hom}_{A}(V, U)$, and a morphism from $(U, g, V)$ to another object $\left(U^{\prime}, g^{\prime}, V^{\prime}\right)$ is a pair $(\phi, \psi) \in \operatorname{Hom}_{A}\left(U, U^{\prime}\right) \times \operatorname{Hom}_{A}\left(V, V^{\prime}\right)$ such that $\psi g=g^{\prime} \phi$.

Lemma 7.5. Let $A$ be a hereditary $R$-order and assume $R$ is a complete DVR. Then every object in $\operatorname{Mor}(\mathscr{P}(A))$ is a direct sum of finitely many objects $M$ such that $\operatorname{End}_{\operatorname{Mor}(\mathscr{P}(A))}(M) \otimes_{R} F$ is a division ring.

Proof. We shall make use of the structure theory of hereditary orders over complete DVRs. The facts that we now cite are taken from [6, $\S \S 2.2,2.3]$; for an extensive discussion, see [31, §39]. Firstly, $A$ is a product of hereditary orders $A=\prod_{i} A_{i}$ such that $\left(A_{i}\right)_{F}$ is simple artinian for all $i$. Working in each factor separately, we may assume that $A_{F}$ is simple and write $A_{F}=\mathrm{M}_{\ell}(D)$ with $D$ a division $F$ algebra. In this case, the (additive) valuation of $F$ extends uniquely to a discrete valuation $\nu_{D}$ on $D$. We let $\mathcal{O}_{D}$ denote the valuation ring of $\nu_{D}$ in $D$, and we let $\mathfrak{m}_{D}$ denote its maximal ideal. Every f.g. projective $A$-module is a direct sum of finitely many indecomposable modules, and there are finitely many isomorphism classes of the latter. Let $P_{1}, \ldots, P_{t}$ be representatives for these classes. After a suitable relabeling, one can identify $\operatorname{Hom}_{A}\left(P_{i}, P_{j}\right)$ with $\mathcal{O}_{D}$ if $i \leq j$, or $\mathfrak{m}_{D}$ if $i>j$, and this identification turns composition into multiplication in $D$.

Now consider $(U, g, V) \in \operatorname{Mor}(\mathscr{P}(A))$ and write $U=\bigoplus_{i=1}^{m} P_{r_{i}}$ and $V=\bigoplus_{j=1}^{n} P_{s_{j}}$ with $r_{1} \leq \cdots \leq r_{m}$ and $s_{1} \leq \cdots \leq s_{n}$. Using the identification of $\operatorname{Hom}_{A}\left(P_{r_{i}}, P_{s_{j}}\right)$ with $\mathcal{O}_{D}$ or $\mathfrak{m}_{D}$, we may regard $g$ as an $n \times m$ matrix with entries in $\mathcal{O}_{D}$; the $(j, i)$-entry lives in $\operatorname{Hom}_{A}\left(P_{r_{i}}, P_{s_{j}}\right)$. We likewise regard $\operatorname{End}_{A}(U)$ as a subring of $\mathrm{M}_{m}\left(\mathcal{O}_{D}\right)$ and $\operatorname{End}_{A}(V)$ as a subring of $\mathrm{M}_{n}\left(\mathcal{O}_{D}\right)$. The assumption $r_{1} \leq \cdots \leq r_{m}$ guarantees that $\operatorname{End}_{A}(U)$ contains

$$
E_{m}:=\left[\begin{array}{cccc}
\mathcal{O}_{D} & \mathfrak{m}_{D} & \ldots & \mathfrak{m}_{D} \\
\mathcal{O}_{D} & \mathcal{O}_{D} & \ddots & \vdots \\
\vdots & & \ddots & \mathfrak{m}_{D} \\
\mathcal{O}_{D} & \ldots & \ldots & \mathcal{O}_{D}
\end{array}\right] \subseteq \mathrm{M}_{m}\left(\mathcal{O}_{D}\right)
$$

and, defining $E_{n} \subseteq \mathrm{M}_{n}\left(\mathcal{O}_{D}\right)$ similarly, we likewise have $E_{n} \subseteq \operatorname{End}_{A}(V)$.

Using elementary row and column operations, it is easy to see that there exist $w \in E_{m}^{\times}$and $v \in E_{n}^{\times}$such that $v g w$ is a monomial matrix, i.e. it contains at most one non-zero entry in every row and every column. (Briefly, writing $g=\left(g_{j i}\right)_{j, i}$, choose $\left(j_{0}, i_{0}\right)$ such that $g_{j_{0} i_{0}}$ is of minimal valuation among the entries of $g$ and such that every $(j, i) \neq\left(j_{0}, i_{0}\right)$ with $i \leq i_{0}, j \leq j_{0}$ satisfies $\nu_{D}\left(g_{j i}\right)>\nu_{D}\left(g_{j_{0} i_{0}}\right)$. Now use elements of $E_{n}^{\times}$and $E_{m}^{\times}$to annihilate the entries of the $j_{0}$-th row and $i_{0}$-th column of $g$ except the $\left(j_{0}, i_{0}\right)$ entry. Delete the $j_{0}$-th row and $i_{0}$-th column and proceed by induction.) Replacing the decompositions $U=\bigoplus_{i} P_{r_{i}}, V=\bigoplus_{j} P_{s_{j}}$ with $U=\bigoplus_{i} w^{-1} P_{r_{i}}, V=\bigoplus_{j} v P_{s_{j}}$, we find that $g$ is represented by a monomial matrix. This means that $(U, g, V)$ decomposes as a direct sum of morphisms of the form $\left(P_{i}, 0,0\right),\left(0,0, P_{j}\right)$ and $\left(P_{i}, f, P_{j}\right)$ with $f \neq 0$. Recalling that $\operatorname{End}_{A}\left(P_{i}\right) \cong$ $\mathcal{O}_{D}$ and $\operatorname{End}_{A}\left(P_{i}, P_{j}\right) \subseteq \mathcal{O}_{D}$, one easily checks that the endomorphism rings of $\left(P_{i}, 0,0\right),\left(0,0, P_{j}\right)$ and $\left(P_{i}, f, P_{j}\right)(f \neq 0)$ are isomorphic to $\mathcal{O}_{D}$ as $R$-algebras. Since $\left(\mathcal{O}_{D}\right)_{F}=D$, the lemma follows. 
Proof of Theorem 7.4. We follow the same argument as in the proof of Theorem 7.1, but use the hermitian category constructed in [5, §3] instead of the one in [7, §4].2 This category is $\operatorname{Mor}(\mathscr{P}(A))$ endowed with a certain hermitian structure. By [5, Th. 1], there is an equivalence between the category of (arbitrary) hermitian forms over $(A, \sigma)$ and the category of unimodular 1-hermitian forms over $\operatorname{Mor}(\mathscr{P}(A))$. One can check that this correspondence is compatible with flat scalar extension in the sense of [7, §2.4], either directly, or by arguing as in the proof of [7, Pr. 3.7].

Arguing as in the proof of Theorem 7.1, we are reduced to show that the genus of unimodular 1-hermitian forms over $\left(A^{\prime \prime}, \sigma^{\prime \prime}\right)$ has size 1 , where $A^{\prime \prime}$ is the endomorphism ring of some object in $\operatorname{Mor}(\mathscr{P}(A))$. It is therefore enough to show that the endomorphism ring of every object in $\operatorname{Mor}(\mathscr{P}(A))$ satisfies the assumptions of Theorem 6.2

Let $(U, g, V) \in \operatorname{Mor}(\mathscr{P}(A))$. It is easy to check that for every flat $R$-algebra $T$, there is a canonical isomorphism

$$
\operatorname{End}_{\operatorname{Mor}\left(\mathscr{P}\left(A_{T}\right)\right)}\left(U_{T}, g_{T}, V_{T}\right) \cong \operatorname{End}_{\operatorname{Mor}(\mathscr{P}(A))}(U, g, V) \otimes_{R} T .
$$

Recall further that $A_{S}$ is hereditary by Theorem 6.6. Now, noting that $A_{S}=$ $\prod_{\mathfrak{p} \neq 0} A_{\hat{R}_{\mathfrak{p}}}$, Lemma 7.5 implies that $\left(U_{S}, g_{S}, V_{S}\right)$ can be written as a direct sum $\bigoplus_{i=1}^{t}\left(U_{i}, g_{i}, V_{i}\right)$ in $\operatorname{Mor}\left(\mathscr{P}\left(A_{S}\right)\right)$ such that

$$
\operatorname{End}\left(U_{i}, g_{i}, V_{i}\right) \otimes_{R} F=\operatorname{End}\left(U_{i}, g_{i}, V_{i}\right) \otimes_{S} K
$$

is local for all $i$. Let $e_{i} \in \operatorname{End}\left(U_{S}, g_{S}, V_{S}\right)=\operatorname{End}(U, g, V) \otimes_{R} S$ be the projection onto the summand $\left(U_{i}, g_{i}, V_{i}\right)$. Then the idempotents $e_{1}, \ldots, e_{s}$ satisfy condition (2) of Theorem 6.2 for $A^{\prime \prime}:=\operatorname{End}(U, g, V)$. That $A_{S}^{\prime \prime}$ is semilocal and $A_{K}^{\prime \prime}$ is semiperfect is clear, so we are done.

7C. Cancellation and Springer's Theorem. As an application of the previous results, we now give versions of Witt's Cancellation Theorem and Springer's (weak) Theorem. We assume $2 \in R^{\times}$throughout.

Corollary 7.6 (Cancellation). Let $A$ be a hereditary $R$-order, let $\sigma: A \rightarrow A$ be an $R$-involution and let $u \in \operatorname{Cent} A$ be an element in $A$ satisfying $u^{\sigma} u=1$. Let $(P, f),\left(P^{\prime}, f^{\prime}\right),\left(P^{\prime \prime}, f^{\prime \prime}\right)$ be u-hermitian forms over $(A, \sigma)$. If $f \perp f^{\prime} \cong f \perp f^{\prime \prime}$, then $f^{\prime} \cong f^{\prime \prime}$.

Proof. By Theorem 7.4, it is enough to show that $f_{\hat{R}_{\mathfrak{p}}}^{\prime} \cong f_{\hat{R}_{\mathfrak{p}}}^{\prime \prime}$ for all $\mathfrak{p} \in \operatorname{Spec} R$ (including $\mathfrak{p}=0$ ). Since $f_{\hat{R}_{\mathfrak{p}}} \perp f_{\hat{R}_{\mathfrak{p}}}^{\prime} \cong f_{\hat{R}_{\mathfrak{p}}} \perp f_{\hat{R}_{\mathfrak{p}}}^{\prime \prime}$, it is enough to show that cancellation holds for (arbitrary) $u$-hermitian forms over finite $\hat{R}_{\mathfrak{p}}$-algebras, and this was shown in [5, Th. 3] (see also [7, $§ 5 \mathrm{~A}$ ] for further results of this kind).

Remark 7.7. Over general semilocal rings, cancellation fails even in the unimodular case; see [23].

For the next result, recall that an étale extension of $R$ is a faithfully flat finitely presented commutative $R$-algebra $R^{\prime}$ such that for every $\mathfrak{p} \in \operatorname{Spec} R$ (including $\mathfrak{p}=0), R_{k(\mathfrak{p})}^{\prime}:=R^{\prime} \otimes_{R} k(\mathfrak{p})$ is a finite product of separable field extensions of $k(\mathfrak{p})$ $\left(\right.$ where $\left.k(\mathfrak{p})=R_{\mathfrak{p}} / \mathfrak{p}_{\mathfrak{p}}\right)$. This is equivalent to $\operatorname{Spec} R^{\prime} \rightarrow \operatorname{Spec} R$ being an étale cover.

\footnotetext{
${ }^{2}$ One can also take the hermitian categories constructed in [7] §3] and [10, §3]. The hermitian category of [5, §3] embeds as a full subcategory inside these hermitian categories, which are isomorphic in our setting [7, Rm. 3.2].
} 
For example, all Galois extensions of $R$ are étale, and the algebra $\prod_{0 \neq \mathfrak{p} \in \operatorname{Spec} R} R_{\mathfrak{p}}$ is also étale over $R$. The rank of $R^{\prime}$ is a function $\operatorname{rank}\left(R^{\prime}\right): \operatorname{Spec} R \rightarrow \mathbb{Z}$ taking $\mathfrak{p}$ to $\operatorname{dim}_{k(\mathfrak{p})} R_{k(\mathfrak{p})}^{\prime}$. This function is constant if $R^{\prime}$ is a finitely generated projective $R$-module. We say that $R^{\prime}$ has odd rank over $R$ if $\operatorname{rank}\left(R^{\prime}\right)$ attains only odd values.

Corollary 7.8 (Springer's Theorem). Let A, $\sigma, u$ be as in Corollary 7.6, and let $R^{\prime}$ be an étale $R$-algebra of odd rank. Let $(P, f),\left(P^{\prime}, f^{\prime}\right)$ be u-hermitian forms over $(A, \sigma)$. If $f_{R^{\prime}} \cong f_{R^{\prime}}^{\prime}$, then $f \cong f^{\prime}$.

Proof. It is easy to check that if $T$ is any commutative $R$-algebra, then $R_{T}^{\prime}$ is étale of odd rank over $T$. Thus, as in the proof of Corollary 17.6, it is enough to prove the theorem in case $R$ is a complete discrete valuation ring.

Now, as in the proof of Theorem 7.4, we may reduce to unimodular 1-hermitian forms over an involutary $R$-order (not-necessarily hereditary), so assume henceforth $f$ and $f^{\prime}$ are unimodular. Write $k=R / \operatorname{Jac}(R)$ and $k^{\prime}=R^{\prime} \otimes_{R} k$. Since $R$ is complete in the $\operatorname{Jac}(R)$-adic topology, it is enough to prove $f_{k} \cong f_{k}^{\prime}$ ([30, Th. 2.2(2)] or [24, Th. II.4.6.1]).

Note that $f_{R^{\prime}} \cong f_{R^{\prime}}$ implies $f_{k^{\prime}} \cong f_{k^{\prime}}^{\prime}$. Since $k^{\prime}$ is étale over $k, k^{\prime}$ is a finite product of field extensions of $k$. Since $k^{\prime}$ has odd rank over $k$, at least one of these fields, denote it $k_{0}$, has odd dimension over $k$. Now, $f_{k_{0}} \cong f_{k_{0}}^{\prime}$, so by [9, Prp. 1.2, Th. 2.1] (Springer's Theorem for quadratic spaces over finite-dimensional involutary algebras), $f_{k} \cong f_{k}^{\prime}$ and we are done.

Remark 7.9. Using similar ideas, one can prove Corollaries 7.6 and 7.8 for systems of sesquilinear forms in the setting of Theorem 7.2 .

\section{APpendix A. IsOMETRY GROUPs AS GROUP SCHEMES}

This appendix shows that under certain assumptions, isometry groups can be realized as faithfully flat smooth affine group schemes. The arguments presented are almost entirely due to Mathieu Huruguen and we thank him for his contribution.

Throughout, $R$ is a commutative ring and $(A, \sigma, u, \Lambda)$ is a unitary $R$-algebra (see 1C). We further assume that $A$ is finitely generated and projective over $R$ and $\Lambda$ is a summand of $A$. Recall that Comm- $R$ denotes the category of commutative $R$-algebras. The acronym "fppf" stands for "finitely presented faithfully flat".

Proposition A.1. Let $(P,[f]),\left(P^{\prime},\left[f^{\prime}\right]\right) \in \mathrm{UQ}^{u, \Lambda}(A, \sigma)$. Denote by $\operatorname{Iso}\left([f],\left[f^{\prime}\right]\right)$ the set of isometries from $(P,[f])$ to $\left(P^{\prime},\left[f^{\prime}\right]\right)$. Then the functor

$$
S \mapsto \operatorname{Iso}\left(\left[f_{S}\right],\left[f_{S}^{\prime}\right]\right): \text { Comm- } R \rightarrow \text { Set }
$$

is the functor of points of a smooth finitely presented affine scheme over Spec $R$, denoted $\mathbf{I s o}\left([f],\left[f^{\prime}\right]\right)$. Furthermore, if for every $\mathfrak{p} \in \operatorname{Spec} R$, there is a field extension $L / k(\mathfrak{p})$ such that $P_{L} \cong P_{L}^{\prime}$ (as $A_{L}$-modules), then $\mathbf{I s o}\left([f],\left[f^{\prime}\right]\right)$ is faithfully flat over $\operatorname{Spec} R$, and there exists an fppf $R$-algebra $S$ such that $\left[f_{S}\right] \cong\left[f_{S}^{\prime}\right]$.

Proof. Choose $B, Q, Q^{\prime} \in \mathscr{P}(R)$ such that $A \oplus B, P \oplus Q, P^{\prime} \oplus Q^{\prime}$ are free, and fix isomorphisms $A \oplus B \cong R^{k}, P \oplus Q \cong R^{m}, P^{\prime} \oplus Q^{\prime} \cong R^{n}$ (elements of $R^{k}, R^{m}, R^{n}$ are viewed as column vectors). For every $S \in \operatorname{Comm}-R$, we embed Iso([ $\left.\left.f_{S}\right],\left[f_{S}^{\prime}\right]\right)$ in $\mathrm{M}_{n \times m}(S) \times \mathrm{M}_{m \times n}(S) \cong S^{2 m n}$ via

$\phi \mapsto\left(\phi \oplus 0, \phi^{-1} \oplus 0\right) \in \operatorname{Hom}_{S}\left(S^{m}, S^{n}\right) \times \operatorname{Hom}\left(S^{n}, S^{m}\right) \cong \mathrm{M}_{n \times m}(S) \times \mathrm{M}_{m \times n}(S)$. 
We shall give finitely many polynomial equations on $R^{2 m n} \cong \mathrm{M}_{m \times n}(R) \times \mathrm{M}_{n \times m}(R)$ such that for every $S \in$ Comm- $R$, their zero locus in $S^{2 m n}$ is Iso $\left(\left[f_{S}\right],\left[f_{S}^{\prime}\right]\right)$. This will prove that the functor $S \mapsto \operatorname{Iso}\left(\left[f_{S}\right],\left[f_{S}^{\prime}\right]\right)$ is the points functor of the closed subscheme of $\mathbb{A}_{R}^{2 m n}$ defined by these equations.

Let $E \in \mathrm{M}_{m \times m}(R)$ and $E^{\prime} \in \mathrm{M}_{n \times n}(R)$ be the matrices corresponding to the projections $R^{m}=P \oplus Q \rightarrow P$ and $R^{n}=P^{\prime} \oplus Q^{\prime} \rightarrow P^{\prime}$, respectively. Likewise, let $G \in \mathrm{M}_{k \times k}(R)$ be a matrix corresponding to some projection of $R^{k}=A \oplus B$ onto the summand $\Lambda$. Let $a_{1}, \ldots, a_{r} \in A$ be a set of generators of $A$ as an $R$-algebra. For each $1 \leq i \leq r$, let $A_{i} \in \mathrm{M}_{m \times m}(R)$ be the matrix corresponding to the map $x \oplus y \mapsto x a_{i} \oplus 0: P \oplus Q \rightarrow P \oplus Q$. Define $A_{1}^{\prime}, \ldots, A_{r}^{\prime} \in \mathrm{M}_{n \times n}(R)$ similarly, by replacing $P, Q$ with $P^{\prime}, Q^{\prime}$. Next, extend $\tilde{h}_{f}: P \times P \rightarrow A$ to an $R$-bilinear function $H: R^{m} \times R^{m} \rightarrow R^{k}$ by $H\left(x_{1} \oplus y_{1}, x_{2} \oplus y_{2}\right)=\tilde{h}_{f}\left(x_{1}, x_{2}\right) \oplus 0$ (where $x_{1}, x_{2} \in P$, $\left.y_{1}, y_{2} \in Q\right)$. There are matrices $H_{1}, \ldots, H_{k} \in \mathrm{M}_{m \times m}(R)$ such that $H(x, y)=$ $\left(x^{T} H_{1} y, \ldots, x^{T} H_{k} y\right)$ for all $x, y \in R^{m}$. Similarly, define $H_{1}^{\prime}, \ldots, H_{k}^{\prime} \in \mathrm{M}_{n \times n}(R)$ by replacing $h_{f}$ with $h_{f^{\prime}}$. We repeat this procedure with $f$ and $f^{\prime}$ in place of $h_{f}$ and $h_{f^{\prime}}$ to get matrices $F_{1}, \ldots, F_{k} \in \mathrm{M}_{m \times m}(R), F_{1}^{\prime}, \ldots, F_{k}^{\prime} \in \mathrm{M}_{n \times n}(R)$. Finally, for a matrix $X$, denote by $d(X)$ the row vector consisting of the diagonal entries of $X$. It is now routine to check that a pair of matrices $(X, Y) \in \mathrm{M}_{m \times n}(S) \times \mathrm{M}_{n \times m}(S)$ lies in Iso $\left(\left[f_{S}\right],\left[f_{S}^{\prime}\right]\right)$ if and only if the following matrix equations, which are defined over $R$, are satisfied:

(1) $E^{\prime} X E=X$ ("there is $\phi \in \operatorname{Hom}_{R}\left(P, P^{\prime}\right)$ such that $X=\phi \oplus 0$ "),

(2) $E Y E^{\prime}=Y, X Y=E^{\prime}, Y X=E$ (" $\phi$ is invertible, $Y=\phi^{-1} \oplus 0$ "),

(3) $X A_{1}=A_{1}^{\prime} X, \ldots, X A_{r}=A_{r}^{\prime} X$ (" $\phi$ is $A$-linear"),

(4) $X^{T} H_{1}^{\prime} X=H_{1}, \ldots, X^{T} H_{k}^{\prime} X=H_{k}\left(" \tilde{h}_{f^{\prime}}(\phi x, \phi y)=\tilde{h}_{f}(x, y)\right.$ "),

(5) $G \cdot D(X)=D(X)$, where $D(X)$ is the matrix whose rows are $d\left(X^{T} F_{1}^{\prime} X-F_{1}\right), \ldots, d\left(X^{T} F_{k}^{\prime} X-F_{k}\right)\left(" \tilde{f}^{\prime}(\phi x, \phi x)-\tilde{f}(x, x) \in \Lambda^{\prime}\right)$.

We now show that Iso( $\left([f],\left[f^{\prime}\right]\right)$ is smooth over $\operatorname{Spec} R$. Since $\mathbf{I s o}\left([f],\left[f^{\prime}\right]\right)$ is of finite presentation, it is enough to check that Iso $\left([f],\left[f^{\prime}\right]\right)$ is formally smooth (see [19, Th. 17.5.1]). Let $S$ be a commutative $R$-algebra and let $I \triangleleft S$ be an ideal with $I^{2}=0$. We need to show that

$$
\xi \mapsto \bar{\xi}: \operatorname{Iso}\left(\left[f_{S}\right],\left[f_{S}^{\prime}\right]\right) \rightarrow \operatorname{Iso}\left(\left[f_{S / I}\right],\left[f_{S / I}^{\prime}\right]\right)
$$

is surjective. This is a well-known argument (e.g. see [30, Th. 2.2]); we recall it for the sake of completeness. Let $\phi \in \operatorname{Iso}\left(\left[f_{S / I}\right],\left[f_{S / I}^{\prime}\right]\right)$. Since $P$ and $P^{\prime}$ are projective over $A$, taking Hom-sets and dualizing with respect to $A$ commute with scalar extension (up to natural isomorphism; cf. Lemma 1.2). Thus, there exists $\psi \in \operatorname{Hom}_{A_{S}}\left(P_{S}, P_{S}^{\prime}\right) \cong \operatorname{Hom}_{A}\left(P, P^{\prime}\right)_{S}$ whose image in $\operatorname{Hom}_{A_{S / I}}\left(P_{S / I}, P_{S / I}^{\prime}\right) \cong$ $\operatorname{Hom}_{A}\left(P, P^{\prime}\right)_{S / I}$ is $\phi$. Since $\phi$ is an isometry, we have $\phi^{*} f_{S / I}^{\prime} \phi-f_{S / I} \in \Lambda_{P_{S / I}}$. By Proposition 1.3. the map $\Lambda_{P_{S}} \otimes_{S} S / I \rightarrow \Lambda_{P_{S / I}}$ is surjective, and hence there is $r \in \Lambda_{P_{S}}$ such that

$g:=\psi^{*} f_{S}^{\prime} \psi-f_{S}-r \in \operatorname{ker}\left(\operatorname{Hom}_{A}\left(P, P^{\prime}\right)_{S} \rightarrow \operatorname{Hom}_{A}\left(P, P^{\prime}\right)_{S / I}\right)=I \cdot \operatorname{Hom}_{A}\left(P, P^{\prime}\right)_{S}$.

Let $c:=-\left(h_{f_{S}^{\prime}}\right)^{-1}\left(\psi^{*}\right)^{-1} g$ and $\xi=\psi+c$ (note that $h_{f_{S}^{\prime}}=f_{S}^{\prime}+f_{S}^{\prime *} \omega$ is invertible because $\left[f^{\prime}\right]$ is unimodular). Then $\bar{\xi}=\phi$ (because $\left.c \in I \cdot \operatorname{Hom}_{A}\left(P, P^{*}\right)_{S}\right)$, and it straightforward to check that

$$
\xi^{*} f_{S}^{\prime} \xi-f_{S}=r+\left(c^{*} f_{S}^{\prime} \psi\right)-\left(c^{*} f_{S}^{\prime} \psi\right)^{*} \omega \in \Lambda_{P_{S}}
$$


(use the fact that $\left.g=-\psi^{*}\left(f_{S}^{\prime}+f_{S}^{\prime *} \omega\right) c\right)$. Thus, $\xi$ is an isometry from $\left[f_{S}\right]$ to $\left[f_{S}^{\prime}\right]$. That $\xi$ is invertible is shown by similar means and is left to the reader.

To finish, assume that for every $\mathfrak{p} \in \operatorname{Spec} R$, there is a field extension $L / k(\mathfrak{p})$ with $P_{L} \cong P_{L}^{\prime}$, and write Iso $\left([f],\left[f^{\prime}\right]\right)=\operatorname{Spec} S$ for suitable $S \in$ Comm- $R$. Since $\operatorname{Iso}\left([f],\left[f^{\prime}\right]\right)(S)=\operatorname{Iso}\left(\left[f_{S}\right],\left[f_{S}^{\prime}\right]\right)$ is tautologically non-empty, it is enough to show that Spec $S \rightarrow \operatorname{Spec} R$ is faithfully flat. Since this morphism is smooth, and in particular flat, it is left to check that $\operatorname{Spec} S \rightarrow \operatorname{Spec} R$ has non-empty fibers. Indeed, let $\mathfrak{p} \in \operatorname{Spec} R$, and choose an algebraically closed field $L \supseteq k(\mathfrak{p})$ such that $P_{L} \cong P_{L}^{\prime}$. By $[30,3.4(3)]$ (for instance), the latter implies $\left[f_{L}\right] \cong\left[f_{L}^{\prime}\right]$, so Iso $\left([f],\left[f^{\prime}\right]\right)(L) \neq \emptyset$. Thus, the fiber $\operatorname{Spec} S \times \operatorname{Spec} R \operatorname{Spec} k(\mathfrak{p})$ has an $L$-point.

For $(P,[f]) \in \mathrm{UQ}^{u, \Lambda}(A, \sigma)$, we write $\mathbf{O}([f])=\mathbf{I s o}([f],[f])$. The proposition shows that $\mathbf{O}([f])$ is a smooth fppf affine scheme over $\operatorname{Spec} R$.

Remark A.2. (i) The proof of the proposition also shows that isometry groups of non-unimodular forms can be regarded as affine schemes over $\operatorname{Spec} R$, but they are not flat in general.

(ii) The scheme $\mathbf{O}([f])$ is a closed subscheme of $\operatorname{End}_{A}(P)$, the scheme corresponding to $\operatorname{End}_{A}(P)$ (cf. Proposition 2.3): Keeping the notation of the proof, embed Iso $\left(\left[f_{S}\right],\left[f_{S}\right]\right)$ in $\operatorname{End}_{S}(P \oplus Q) \cong \mathrm{M}_{m}(S) \cong S^{m^{2}}$ via $\phi \mapsto \phi \oplus 0$. Then $\operatorname{End}_{A_{S}}\left(P_{S}\right)$ is the zero locus of equations $(1)$ and $(3)$ above, while $\operatorname{Iso}\left(\left[f_{S}\right],\left[f_{S}\right]\right)$ is the zero locus of equations (1) and (3)-(5), hence our claim. (Note that equation (4) implies that the isometry $\phi$ corresponding to $X$ is invertible: Since $\phi^{*} h_{f} \phi=h_{f}$ and $[f]$ is unimodular, $\phi$ is invertible on the left, and hence so is $\phi \oplus \operatorname{id}_{Q} \in \operatorname{End}(P \oplus Q) \cong \mathrm{M}_{m}(S)$. Now, easy determinant considerations imply $\phi \oplus \operatorname{id}_{Q}$ is invertible on the right, so $\phi$ is invertible.)

\section{REFERENCES}

[1] Maurice Auslander and Oscar Goldman, The Brauer group of a commutative ring, Trans. Amer. Math. Soc. 97 (1960), 367-409, DOI 10.2307/1993378. MR0121392

[2] Maurice Auslander and Oscar Goldman, Maximal orders, Trans. Amer. Math. Soc. 97 (1960), 1-24, DOI 10.2307/1993361. MR0117252

[3] Paul Balmer, Witt groups, Handbook of $K$-theory. Vol. 1, 2, Springer, Berlin, 2005, pp. 539576, DOI 10.1007/978-3-540-27855-9_11. MR2181829

[4] Hyman Bass, Unitary algebraic K-theory, Algebraic K-theory, III: Hermitian K-theory and geometric applications (Proc. Conf., Battelle Memorial Inst., Seattle, Wash., 1972), Lecture Notes in Math., vol. 343, Springer, Berlin, 1973, pp. 57-265. MR0371994

[5] Eva Bayer-Fluckiger and Laura Fainsilber, Non-unimodular Hermitian forms, Invent. Math. 123 (1996), no. 2, 233-240, DOI 10.1007/s002220050024. MR1374198

[6] Eva Bayer-Fluckiger and Uriya A. First, Rationally isomorphic hermitian forms and torsors of some non-reductive groups, Adv. Math. 312 (2017), 150-184, DOI 10.1016/j.aim.2017.03.012. MR.3635808

[7] Eva Bayer-Fluckiger, Uriya A. First, and Daniel A. Moldovan, Hermitian categories, extension of scalars and systems of sesquilinear forms, Pacific J. Math. 270 (2014), no. 1, 1-26, DOI 10.2140/pjm.2014.270.1. MR3245846

[8] Eva Bayer-Fluckiger, Cherry Kearton, and Stephen M. J. Wilson, Hermitian forms in additive categories: finiteness results, J. Algebra 123 (1989), no. 2, 336-350, DOI 10.1016/00218693(89)90050-1. MR.1000491

[9] Eva Bayer-Fluckiger and Hendrick W. Lenstra Jr., Forms in odd degree extensions and self-dual normal bases, Amer. J. Math. 112 (1990), no. 3, 359-373, DOI 10.2307/2374746. MR 1055648 
[10] Eva Bayer-Fluckiger and Daniel Arnold Moldovan, Sesquilinear forms over rings with involution, J. Pure Appl. Algebra 218 (2014), no. 3, 417-423, DOI 10.1016/j.jpaa.2013.06.012. MR.3124208

[11] Siegfried Bosch, Werner Lütkebohmert, and Michel Raynaud, Néron models, Ergebnisse der Mathematik und ihrer Grenzgebiete (3) [Results in Mathematics and Related Areas (3)], vol. 21, Springer-Verlag, Berlin, 1990. MR.1045822

[12] Nicolas Bourbaki, Lie groups and Lie algebras. Chapters 4-6, Elements of Mathematics (Berlin), Springer-Verlag, Berlin, 2002. Translated from the 1968 French original by Andrew Pressley. MR 1890629

[13] Jean-Louis Colliot-Thélène and Manuel Ojanguren, Espaces principaux homogènes localement triviaux (French), Inst. Hautes Études Sci. Publ. Math. 75 (1992), 97-122. MR1179077

[14] Brian Conrad, Weil and Grothendieck approaches to adelic points, Enseign. Math. (2) 58 (2012), no. 1-2, 61-97, DOI 10.4171/LEM/58-1-3. MR2985010

[15] Otto Endler, Valuation theory, Springer-Verlag, New York-Heidelberg, 1972. To the memory of Wolfgang Krull (26 August 1899-12 April 1971); Universitext. MR0357379

[16] Daniel Ferrand and Michel Raynaud, Fibres formelles d'un anneau local noethérien (French), Ann. Sci. École Norm. Sup. (4) 3 (1970), 295-311. MR.0272779

[17] Uriya A. First, Witt's extension theorem for quadratic spaces over semiperfect rings, J. Pure Appl. Algebra 219 (2015), no. 12, 5673-5696, DOI 10.1016/j.jpaa.2015.05.039. MR3390045

[18] Philippe Gille, Torseurs sur la droite affine, Transform. Groups 7 (2002), no. 3, 231-245, DOI 10.1007/s00031-002-0012-3. MR1923972

[19] Alexander Grothendieck, Éléments de géométrie algébrique. IV. Étude locale des schémas et des morphismes de schémas IV, Inst. Hautes Études Sci. Publ. Math. 32 (1967), 361. MR0238860

[20] David Harbater, Julia Hartmann, and Daniel Krashen, Applications of patching to quadratic forms and central simple algebras, Invent. Math. 178 (2009), no. 2, 231-263, DOI 10.1007/s00222-009-0195-5. MR2545681

[21] Hiroaki Hijikata and Kenji Nishida, Bass orders in nonsemisimple algebras, J. Math. Kyoto Univ. 34 (1994), no. 4, 797-837, DOI 10.1215/kjm/1250518887. MR1311621

[22] John S. Kauta, Weak crossed-product orders over valuation rings, J. Algebra 402 (2014), 319-350, DOI 10.1016/j.jalgebra.2013.12.014. MR3160425

[23] Bernhard Keller, A remark on quadratic spaces over noncommutative semilocal rings, Math. Z. 198 (1988), no. 1, 63-71, DOI 10.1007/BF01183039. MR.938029

[24] Max-Albert Knus, Quadratic and Hermitian forms over rings, Grundlehren der Mathematischen Wissenschaften [Fundamental Principles of Mathematical Sciences], vol. 294, SpringerVerlag, Berlin, 1991. With a foreword by I. Bertuccioni. MR.1096299

[25] Max-Albert Knus, Alexander Merkurjev, Markus Rost, and Jean-Pierre Tignol, The book of involutions, American Mathematical Society Colloquium Publications, vol. 44, American Mathematical Society, Providence, RI, 1998. With a preface in French by J. Tits. MR.1632779

[26] Tsit Yuen Lam, Lectures on modules and rings, Graduate Texts in Mathematics, vol. 189, Springer-Verlag, New York, 1999. MR1653294

[27] Yevsey A. Nisnevich, Etale cohomology and arithmetic of semisimple groups, ProQuest LLC, Ann Arbor, MI, 1982. Thesis (Ph.D.)-Harvard University. MR2632405

[28] Yevsey A. Nisnevich, Espaces homogènes principaux rationnellement triviaux et arithmétique des schémas en groupes réductifs sur les anneaux de Dedekind, C. R. Acad. Sci. Paris Sér. I Math. 299 (1984), no. 1, 5-8. MR756297

[29] O. Timothy O'Meara, Introduction to quadratic forms, Classics in Mathematics, SpringerVerlag, Berlin, 2000. Reprint of the 1973 edition. MR1754311

[30] Heinz-Georg Quebbemann, Winfried Scharlau, and Manfred Schulte, Quadratic and Hermitian forms in additive and abelian categories, J. Algebra 59 (1979), no. 2, 264-289, DOI 10.1016/0021-8693(79)90126-1. MR543249

[31] Irving Reiner, Maximal orders, London Mathematical Society Monographs. New Series, vol. 28, The Clarendon Press, Oxford University Press, Oxford, 2003. Corrected reprint of the 1975 original; With a foreword by M. J. Taylor. MR.1972204

[32] Hans J. Reiter, Witt's theorem for noncommutative semilocal rings, J. Algebra 35 (1975), 483-499, DOI 10.1016/0021-8693(75)90061-7. MR0387276

[33] Louis H. Rowen, Ring theory. Vol. I, Pure and Applied Mathematics, vol. 127, Academic Press, Inc., Boston, MA, 1988. MR.940245 
[34] David J. Saltman, Lectures on division algebras, CBMS Regional Conference Series in Mathematics, vol. 94, Published by American Mathematical Society, Providence, RI; on behalf of Conference Board of the Mathematical Sciences, Washington, DC, 1999. MR.1692654

[35] Winfried Scharlau, Quadratic and Hermitian forms, Grundlehren der Mathematischen Wissenschaften [Fundamental Principles of Mathematical Sciences], vol. 270, Springer-Verlag, Berlin, 1985. MR770063

[36] Nguyêñ Quôć Thăńg, On weak approximation in algebraic groups and related varieties defined by systems of forms, J. Pure Appl. Algebra 113 (1996), no. 1, 67-90, DOI 10.1016/00224049(95)00141-7. MR 1411647

[37] Seth Warner, Topological rings, North-Holland Mathematics Studies, vol. 178, North-Holland Publishing Co., Amsterdam, 1993. MR1240057

[38] William C. Waterhouse, Introduction to affine group schemes, Graduate Texts in Mathematics, vol. 66, Springer-Verlag, New York-Berlin, 1979. MR.547117

École Polytechnique Fédérale de Lausanne, CH-1015 Lausanne, Switzerland

Department of Mathematics, University of British Columbia, Vancouver, British Columbia V6T 1Z2, Canada 